\title{
An Optimal Whitening Approach to Linear Multiuser Detection
}

\author{
Yonina C. Eldar, Member, IEEE, and Albert M. Chan, Student Member, IEEE
}

\begin{abstract}
In this paper, we propose a new linear multiuser receiver for synchronous code-division multiple-access (CDMA) systems, referred to as the orthogonal multiuser (OMU) receiver. Unlike the linear minimum mean-squared error (MMSE) receiver, the OMU receiver depends only on the signature vectors and does not require knowledge of the received amplitudes or the channel signal-to-noise ratio (SNR). Several equivalent representations of the receiver are developed with different implications in terms of implementation. In the first, the receiver consists of a decorrelator demodulator followed by an optimal whitening transformation on a space formed by the signatures. In the second, the receiver consists of a bank of correlators with correlating vectors that are projections of a set of orthogonal vectors, and are closest in a least squares sense to the decorrelator vectors and also closest in a least squares sense to the signature vectors. In the third, the receiver consists of a single-user matched filter (MF) followed by an optimal whitening transformation on a space formed by the signatures.

We derive exact and approximate expressions for the probability of bit error, as well as the asymptotic signal-to-interference + noise ratio (SINR) in the large system limit. The analysis suggests that over a wide range of channel parameters the OMU receiver can outperform both the decorrelator and the single-user MF and perform similarly to the linear MMSE receiver, despite not knowing the channel parameters.
\end{abstract}

Index Terms-Code-division multiple access (CDMA), least squares, multiuser detection, normalized tight frame, orthogonalization, subspace whitening, whitening.

\section{INTRODUCTION}

$\mathbf{M}$ ULTIUSER receivers for detection of code-division multiple-access (CDMA) signals try to mitigate the effect of multiple-access interference (MAI) and background noise. These include the optimal multiuser receiver, the linear minimum mean-squared error (MMSE) receiver, the decorrelator, and the matched filter (MF) receiver [1].

Both the optimal receiver and the linear MMSE receiver require knowledge of the channel parameters, namely, the noise level and the received amplitudes of the users' signals. There are many scenarios, however, where these parameters may not

Manuscript received February 1, 2002; revised March 26, 2003. The work of Y. C. Eldar was supported by the Taub Foundation. The work of A. M. Chan was supported in part by a Natural Sciences and Engineering Research Council of Canada Fellowship.

Y. C. Eldar was with the Research Laboratory of Electronics, Massachusetts Institute of Technology, Cambridge, MA 02139 USA. She is now with the Technion-Israel Institute of Technology, Haifa 32000, Israel (e-mail: yonina@ ee.technion.ac.il).

A. M. Chan is with the Research Laboratory of Electronics, Massachusetts Institute of Technology, Cambridge, MA 02139 USA (e-mail: chanal@alum. mit.edu).

Communicated by D. N. C. Tse, Associate Editor for Communications.

Digital Object Identifier 10.1109/TIT.2003.815761 be known or may be changing over time. On the other hand, the MF and the decorrelator receivers are linear receivers that only require knowledge of the signature vectors. The MF optimally compensates for the white noise, but does not exploit the structure of the MAI; the decorrelator optimally rejects the MAI for linearly independent signature vectors, but does not consider the white noise. A difficulty often encountered when using the decorrelator is that the noise component in the receiver output can have a large variance and a covariance structure with a very high dynamic range.

Recently, a new linear multiuser receiver referred to as the orthogonal multiuser (OMU) receiver was proposed as a compromise between the MF and the decorrelator [2], [3]. Like the $\mathrm{MF}$ and the decorrelator, this receiver requires knowledge of the signature vectors only and is, therefore, designed to operate in environments in which the channel parameters are unknown or time varying. The OMU receiver was developed in [2] under the assumption of linearly independent signature vectors. In this paper, we provide a much more thorough development of the OMU receiver and extend the results to include linearly dependent signature vectors. We also develop tools to analyze the performance of the OMU receiver. The analysis, presented in Section VI, strongly suggests that in certain cases this new receiver outperforms the MF and the decorrelator and approaches the performance of the linear MMSE receiver, even though the received amplitudes of the users and the noise variance are unknown.

Three equivalent representations of the new receiver are developed in Section III. In the first, developed in Section III-A, the receiver consists of a decorrelator demodulator followed by an optimal MMSE whitening transformation [4], [3] on a space formed by the signature vectors. This whitening transformation is designed to optimally decorrelate the outputs of the decorrelator prior to detection, and in that way compensate for the noise enhancement of the decorrelator receiver. Specifically, it minimizes the mean square error (MSE) between the vector output of the decorrelator and the output of the whitening transformation, so that distortion to the output vector is minimized under the whitening constraint. In the second, developed in Section III-B, the demodulator consists of a bank of correlators with correlating vectors that are projections of a set of orthogonal vectors, and are closest in a least squares sense to the decorrelator vectors and also closest in a least squares sense to the signature vectors. In the third, developed in Section III-C, the receiver consists of an MF demodulator followed by an optimal MMSE whitening transformation on a space formed by the signature vectors, which minimizes the MSE between the vector output of the MF and the output of the whitening transformation. 
These three representations are mathematically equivalent, but they provide further insight into the OMU receiver and may have different implications in terms of implementation. The properties of the OMU receiver as well as efficient methods for its implementation are summarized in Section IV.

The whitening approach proposed in this paper is different from previous whitening approaches to multiuser detection [5]-[8]. In the decorrelating decision-feedback detector of [5], the components of the output vector of the feedforward filter are indeed whitened, but not according to the criterion used by the OMU demodulator. Rather, the whitening transformation in [5] is chosen in anticipation of a nonlinear feedback loop. A noise-whitening approach is also used in [6], where the whitening has a different context. Unlike the decorrelating decision-feedback detector and the OMU detector, where the whitening is performed across users in the same symbol period, the detector in [6] performs whitening of the power spectrum of the received chip waveform followed by MF detection of the desired user. Though this method results in poorer performance than typical multiuser detectors, it can be applied to a broader set of circumstances, e.g., interferers are not required to be locked and despreaded, and the spreading codes of interferers are not required to be known. The approaches in [7], [8] are similar in concept to the approach in [6].

In Section V, we show that the OMU demodulator maximizes both the total signal-to-interference ratio (SIR) and the total signal-to-noise ratio (SNR) at the output subject to the whitening constraint when the signature vectors have a certain symmetry property called geometric uniformity [9], [10] which holds, for example, for pseudonoise (PN) sequences corresponding to maximal-length shift-register sequences [1], [11]. The OMU demodulator also approximately maximizes both the total output SIR and the total output SNR for nearly orthogonal signature vectors. This provides some additional justification for this receiver.

In Section VI, we evaluate the performance of the OMU receiver. We first derive exact and approximate expressions for the probability of error. We then analyze the asymptotic performance of the receiver in the large system limit when random Gaussian signatures and accurate power control are used. Specifically, we show that the output signal-to-interference + noise ratio (SINR) of the output of the OMU receiver converges to a deterministic limit. Our method of proof can be easily modified to characterize the performance of other multiuser detectors in the large system limit as well [12]. Using these results, we compare the asymptotic performance of the OMU receiver to that of the decorrelator, MF, and linear MMSE receivers [13], [14], [12]. The analysis demonstrates that in the large system limit, the OMU receiver often performs better than both the MF and the decorrelator receivers, which motivates the use of the OMU receiver in scenarios in which the channel parameters are unknown.

\section{PROBlem Formulation}

Before proceeding to a detailed development of the OMU receiver, in this section we summarize some notation and provide a formulation and overview of our problem.

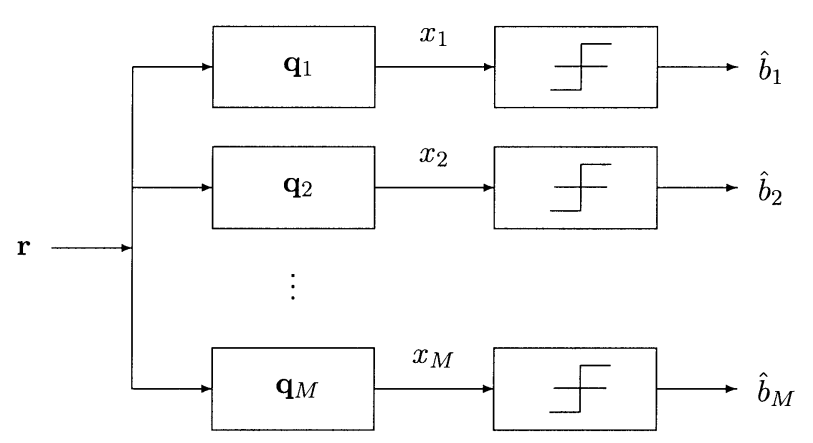

Fig. 1. General linear receiver comprised of a bank of correlators with correlating vectors $\boldsymbol{q}_{m}$ followed by a bank of detectors.

We denote vectors in $\mathbb{C}^{M}$ ( $M$ arbitrary) by boldface lowercase letters, e.g., $\boldsymbol{a}$, where the $m$ th component of $\boldsymbol{a}$ is denoted by $a_{m}$. Matrices in $\mathbb{C}^{N \times M}$ are denoted by boldface uppercase letters, e.g., $\boldsymbol{S}$. The range space and null space of the matrix $\boldsymbol{S}$ are denoted by $\mathcal{R}(\boldsymbol{S})$ and $\mathcal{N}(\boldsymbol{S})$, respectively. $\boldsymbol{I}_{M}$ denotes the $M \times M$ identity matrix, $(\cdot)^{*}$ denotes the Hermitian conjugate of the corresponding matrix, $(\cdot)^{\dagger}$ denotes the Moore-Penrose pseudoinverse [15], and $(\hat{.})$ denotes an optimal vector or matrix. The $m k$ th element and the $m$ th column of a matrix are denoted by $[\cdot]_{m k}$ and $[\cdot]_{m}$, respectively. $P_{\mathcal{U}}$ denotes the orthogonal projection operator onto the subspace $\mathcal{U}$, and $\mathcal{U}^{\perp}$ denotes the orthogonal complement of $\mathcal{U}$. The sign of $a$ is denoted by $\operatorname{sgn}(a)$, and is equal to 1 for $a \geq 0$ and -1 for $a<0$. A prime attached to a random variable or vector denotes the variable or vector with the conditional mean given the transmitted data subtracted, e.g., $\boldsymbol{a}^{\prime}=\boldsymbol{a}-E(\boldsymbol{a} \mid \boldsymbol{b})$, where $\boldsymbol{b}$ is the vector of transmitted data.

Consider an $M$-user white Gaussian synchronous CDMA system where each user transmits information by modulating a signature sequence. The discrete-time model for the received signal $\boldsymbol{r}$ is given by

$$
r=S A b+n
$$

where $\boldsymbol{S}=\left[\boldsymbol{s}_{1}\left|\boldsymbol{s}_{2}\right| \cdots \mid \boldsymbol{s}_{M}\right]$ is the $N \times M$ matrix of signatures with $\boldsymbol{s}_{m} \in \mathbb{C}^{N}$ being the signature vector of the $m$ th user, $\boldsymbol{A}=\operatorname{diag}\left(A_{1}, \ldots, A_{M}\right)$ is the matrix of received amplitudes with $A_{m}>0$ being the amplitude of the $m$ th user's signal, $\boldsymbol{b}=\left[b_{1}, b_{2}, \ldots, b_{M}\right]^{*}$ is the data vector with $b_{m}$ being the $m$ th user's transmitted symbol, and $\boldsymbol{n}$ is a noise vector whose elements are independent $\mathcal{C N}\left(0, \sigma^{2}\right)$. We assume that all data vectors are equally likely with covariance $\boldsymbol{I}_{M}$. For concreteness, we also assume that $b_{m} \in\{1,-1\}$.

Based on the observed signal $\boldsymbol{r}$, we design a linear receiver to detect the information transmitted by each user, which can be implemented using a bank of correlators, as depicted in Fig. 1. We restrict our attention to receivers that do not require knowledge of the received amplitudes $A_{m}$ or the noise level $\sigma^{2}$. The received signal $\boldsymbol{r}$ is cross-correlated with $M$ vectors $\boldsymbol{q}_{m} \in \mathbb{C}^{N}$ so that $x_{m}=\boldsymbol{q}_{m}^{*} \boldsymbol{r}$, where the vectors $\boldsymbol{q}_{m}$ are to be determined and are functions of the signature vectors only. The $m$ th user's bit is then detected as $\hat{b}_{m}=\operatorname{sgn}\left(x_{m}\right)$. Since the noiseless vector $\boldsymbol{S} \boldsymbol{A} \boldsymbol{b}$ lies in the subspace $\mathcal{U}$ spanned by the signature vectors $\boldsymbol{s}_{m}$, we also assume that the correlating vectors $\boldsymbol{q}_{m}$ lie in $\mathcal{U}$.

If we choose the vectors $\boldsymbol{q}_{m}=\boldsymbol{s}_{m}$ in Fig. 1, then the resulting demodulator is equivalent to the single-user MF demodulator 


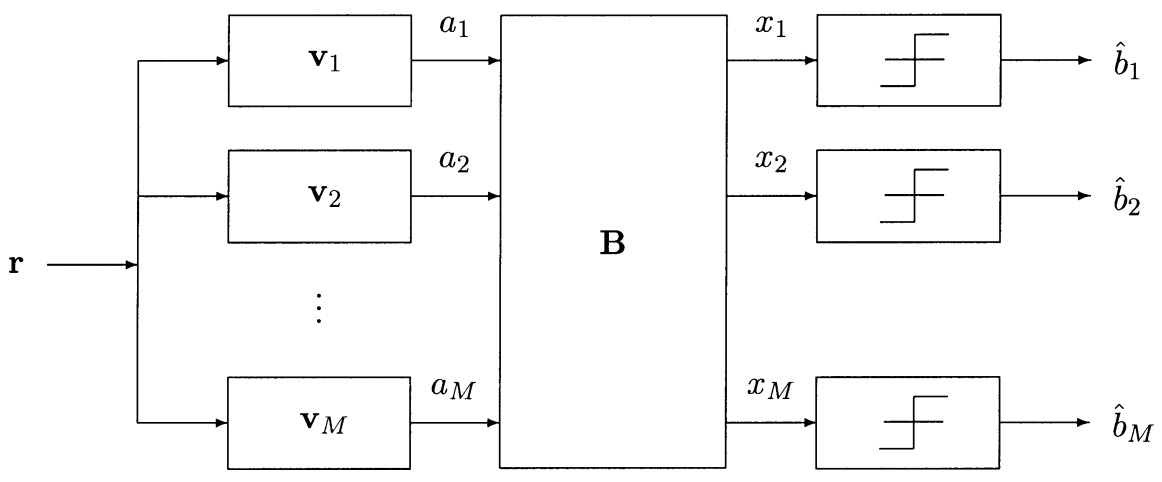

Fig. 2. Alternative representation of Fig. 1 in terms of a linear transformation of the decorrelator outputs. An additional representation can be obtained by replacing the decorrelator vectors $\boldsymbol{v}_{m}$ by the signature vectors $\boldsymbol{s}_{m}$, and by changing the linear transformation $\boldsymbol{B}$ accordingly.

[1]. Choosing the vectors $\boldsymbol{q}_{m}=\boldsymbol{v}_{m}$, where $\boldsymbol{v}_{m}$ are the columns of $\boldsymbol{V}=\left(\boldsymbol{S}^{\dagger}\right)^{*}=\boldsymbol{S}\left(\boldsymbol{S}^{*} \boldsymbol{S}\right)^{\dagger}$, leads to the decorrelator receiver, introduced by Lupas and Verdú [16]. When the signature vectors are linearly independent, the decorrelator optimally rejects the MAI. For linearly dependent signature vectors, which is the case, for example, when the number of users $M$ is larger than the signature length $N$, the decorrelator no longer rejects the MAI. In either case, the inverse operation of the decorrelator may enhance the white noise, resulting in degraded performance. Indeed, the noise component at the output of the decorrelator often has a large variance and a covariance structure with a very high dynamic range.

To develop the OMU receiver, we note that a bank of correlators as depicted in Fig. 1 with vectors $\boldsymbol{q}_{m} \in \mathcal{U}$ is equivalent to a demodulator with correlating vectors that $\operatorname{span} \mathcal{U}$, followed by an appropriate linear transformation on the demodulator outputs. In particular, the receiver of Fig. 1 is equivalent to the receiver of Fig. 2, where $\boldsymbol{v}_{m}$ are the decorrelator vectors and the transformation $\boldsymbol{B}$ is constructed from the decorrelator vectors $\boldsymbol{v}_{m}$ and the correlating vectors $\boldsymbol{q}_{m}$ of Fig. 1. Specifically, with $\boldsymbol{Q}$ denoting the matrix of columns $\boldsymbol{q}_{m}$, the vector output $\boldsymbol{x}$ of Fig. 1 is equal to $\boldsymbol{Q}^{*} \boldsymbol{r}$, and the vector output of Fig. 2 is equal to $\boldsymbol{B} \boldsymbol{V}^{*} \boldsymbol{r}$. Since the vectors $\boldsymbol{q}_{m}$ lie in $\mathcal{U}, \boldsymbol{Q}=P_{\mathcal{U}} \boldsymbol{Q}=\boldsymbol{V} \boldsymbol{V}^{\dagger} \boldsymbol{Q}$, where we used the fact that from the properties of the pseudoinverse, $V V^{\dagger}=P_{\mathcal{U}}$. We may, therefore, express the output of Fig. 1 as

$$
Q^{*} r=Q^{*}\left(V^{\dagger}\right)^{*} V^{*} r=B V^{*} r
$$

with $\boldsymbol{B}=\left(\boldsymbol{V}^{\dagger} \boldsymbol{Q}\right)^{*}$. In the absence of noise, the decorrelator leads to optimal decisions. Thus, in this case choosing $\boldsymbol{B}=\boldsymbol{I}_{M}$ in the receiver of Fig. 2 will result in an optimal receiver for detecting the transmitted symbols. However, in the presence of noise, the decorrelator does not generally lead to optimal decisions. We may therefore wish to further process the outputs of the decorrelator prior to detection.

We may also choose the correlating vectors of Fig. 2 to be equal to the signature vectors, so that the receiver of Fig. 1 can be implemented as an MF demodulator followed by a linear transformation $\boldsymbol{B}=\left(\boldsymbol{S}^{\dagger} \boldsymbol{Q}\right)^{*}$ on the MF outputs. This representation may be of interest since, in many practical receivers, the MF demodulator serves as a front-end whose objective is to obtain a vector representation of the continuous-time received signal. If we choose to linearly process these outputs prior to detection, then the overall demodulator may be expressed as an MF followed by a linear transformation of the MF outputs.

\section{THE OMU DEMODULATOR}

\section{A. Decorrelator Representation of the OMU Demodulator}

As motivated in Section II, we would like to choose the transformation $\boldsymbol{B}$ in Fig. 2 to improve the performance of the decorrelator. It was noted in [13] and [16] that the decorrelator does not generally lead to optimal decisions, since, in general, the noise components in the outputs $a_{m}=\boldsymbol{v}_{m}^{*} \boldsymbol{r}$ of the decorrelator demodulator are correlated. Specifically, from the properties of the pseudoinverse [15], we have that $\boldsymbol{S}^{\dagger} \boldsymbol{S}=P_{\mathcal{N}(\boldsymbol{S})^{\perp}}$, where $P_{\mathcal{N}(\boldsymbol{S}) \perp}$ is the orthogonal projection onto the orthogonal complement of the null space $\mathcal{N}(\boldsymbol{S})$ of $\boldsymbol{S}$, so that the vector output $\boldsymbol{a}$ of the decorrelator is equal to

$$
\boldsymbol{a}=\boldsymbol{V}^{*} \boldsymbol{r}=P_{\mathcal{N}(\boldsymbol{S})^{\perp}} \boldsymbol{A} \boldsymbol{b}+\boldsymbol{V}^{*} \boldsymbol{n} .
$$

The covariance of the noise component $\boldsymbol{V}^{*} \boldsymbol{n}$ in $\boldsymbol{a}$ is equal to the covariance of $\boldsymbol{a}-E(\boldsymbol{a} \mid \boldsymbol{b})$, and is given by

$$
\begin{aligned}
\boldsymbol{C}_{a} & =\sigma^{2} \boldsymbol{V}^{*} \boldsymbol{V}=\sigma^{2}\left(\boldsymbol{S}^{*} \boldsymbol{S}\right)^{\dagger} \boldsymbol{S}^{*} \boldsymbol{S}\left(\boldsymbol{S}^{*} \boldsymbol{S}\right)^{\dagger} \\
& =\sigma^{2} P_{\mathcal{N}(\boldsymbol{S})^{\perp}}\left(\boldsymbol{S}^{*} \boldsymbol{S}\right)^{\dagger}=\sigma^{2}\left(\boldsymbol{S}^{*} \boldsymbol{S}\right)^{\dagger} .
\end{aligned}
$$

From (3) it follows that if the vectors $\boldsymbol{s}_{m}$ are not orthogonal, then the outputs of the decorrelator are correlated. Intuitively it seems plausible that eliminating this common (linear) information may potentially improve the performance of the detector. Furthermore, the pseudoinverse operation in (3) may result in a noise component with a large variance and a spectral shape with a high dynamic range. To improve the performance of the decorrelator receiver it is, therefore, desirable to shape the covariance of the decorrelator outputs prior to detection, and in that way compensate for its noise enhancement properties. Therefore, when the signatures are not orthogonal, we propose to decorrelate the noise components in the outputs of the decorrelator prior to detection, so that we control the dynamic range and spectral shape of the covariance of the receiver output. We emphasize that this task does not require estimating the variance of the noise or the received amplitudes of the users' vectors.

Data whitening arises in a variety of signal processing and communication contexts in which it may be useful to decorrelate a data sequence either prior to subsequent processing, or to 
control the spectral shape after processing. Examples in which data whitening has been used to advantage include enhancing direction-of-arrival algorithms by prewhitening [17], [18]. Rather than choosing the whitening transformation arbitrarily, in the following we suggest choosing an optimal whitening transformation that whitens the decorrelator output and at the same time minimizes the distortion to the unwhitened vector. In Section VI, we analyze the performance of the resulting receiver and show that in many cases this approach does in fact lead to improved performance over the decorrelator and the MF receivers, which provides justification for our method.

When the signature vectors $\boldsymbol{s}_{m}$ are linearly independent, we may decorrelate the outputs of the decorrelator by choosing $\boldsymbol{B}$ in Fig. 2 such that $\boldsymbol{a}$ is whitened. ${ }^{1}$ Specifically, in this case, the covariance matrix $\boldsymbol{C}_{a}=\sigma^{2}\left(\boldsymbol{S}^{*} \boldsymbol{S}\right)^{-1}$ of the noise component in $\boldsymbol{a}$ is invertible and, therefore, there is a whitening transformation $W$ such that the covariance matrix of the noise component of $\boldsymbol{x}=\boldsymbol{W} \boldsymbol{a}$ is given by $\boldsymbol{C}_{x}=\sigma^{2} \boldsymbol{I}_{M}$. Indeed, any transformation $W$ satisfying

$$
\boldsymbol{W}_{a} \boldsymbol{W}^{*}=\sigma^{2} \boldsymbol{W}\left(\boldsymbol{S}^{*} \boldsymbol{S}\right)^{-1} \boldsymbol{W}^{*}=\sigma^{2} \boldsymbol{I}_{M}
$$

is a valid whitening transformation. We then base our detection on $\boldsymbol{x}$, so that the $m$ th user's bit is detected as $\hat{b}_{m}=\operatorname{sgn}\left(x_{m}\right)$.

There are many ways to choose a whitening transformation $\boldsymbol{W}$ satisfying (4), for example using the eigendecomposition or Cholesky factorization of $\boldsymbol{C}_{a}$ [19]. Since the detection is based on the output $x$ of the whitening transformation, we propose choosing the whitening transformation $\boldsymbol{W}$ that results in an output $\boldsymbol{x}$ that is as close as possible in an MSE sense to the output $\boldsymbol{a}$ of the decorrelator so that we minimize the distortion to $\boldsymbol{a}$. Thus, among all possible whitening transformations $\boldsymbol{W}$ we seek the one that minimizes the total MSE given by

$$
\begin{aligned}
\varepsilon_{\mathrm{MSE}} & =\sum_{m=1}^{M} E\left(\left(a_{m}^{\prime}-x_{m}^{\prime}\right)^{2}\right) \\
& =E\left(\left(\boldsymbol{a}^{\prime}-\boldsymbol{x}^{\prime}\right)^{*}\left(\boldsymbol{a}^{\prime}-\boldsymbol{x}^{\prime}\right)\right)
\end{aligned}
$$

where $a_{m}^{\prime}$ and $x_{m}^{\prime}$ are the $m$ th components of $\boldsymbol{a}^{\prime}=\boldsymbol{a}-E(\boldsymbol{a} \mid \boldsymbol{b})$ and $\boldsymbol{x}^{\prime}=\boldsymbol{x}-E(\boldsymbol{x} \mid \boldsymbol{b})$, respectively.

If the signature vectors $\boldsymbol{s}_{m}$ are linearly dependent, then the noise components in the outputs $a_{m}$ are linearly dependent, i.e., they satisfy a deterministic linear relation. Thus, the vector noise output $\boldsymbol{V}^{*} \boldsymbol{n}$ of the decorrelator lies in a subspace $\mathcal{V} \subset \mathbb{C}^{M}$ with probability one (w.p. 1), ${ }^{2}$ which is the range space of $\boldsymbol{S}^{*}$ and is also, from (3), the range space of $\boldsymbol{C}_{a}$. Since the elements of $\boldsymbol{V}^{*} \boldsymbol{n}$ are linearly dependent, the elements of the noise component in $\boldsymbol{x}$, i.e., the elements of $\boldsymbol{W} \boldsymbol{V}^{*} \boldsymbol{n}$, are also linearly dependent and, therefore, cannot be statistically uncorrelated. In other words, the covariance matrix $\boldsymbol{C}_{a}=\sigma^{2}\left(\boldsymbol{S}^{*} \boldsymbol{S}\right)^{\dagger}$ of the noise component in $\boldsymbol{a}$ is not invertible and, therefore, there is no whitening transformation $\boldsymbol{W}$ satisfying (4). Therefore, in this case, we cannot whiten $\boldsymbol{a}$ in the conventional sense. Instead, we propose

\footnotetext{
${ }^{1}$ In this paper, when we refer to the whitening of a random vector $\boldsymbol{a}$, we explicitly mean whitening the noise component in $\boldsymbol{a}$. Equivalently, this corresponds to whitening $\boldsymbol{a}-E(\boldsymbol{a} \mid \boldsymbol{b})$. Similarly, when we say that a random vector $\boldsymbol{x}$ is white we explicitly mean that the noise component in $\boldsymbol{x}$ is white, i.e. the covariance matrix of the noise component in $\boldsymbol{x}, \boldsymbol{x}-E(\boldsymbol{x} \mid \boldsymbol{b})$, is given by $\boldsymbol{C}_{x}=c^{2} \boldsymbol{I}$ for some $c \neq 0$.

${ }^{2}$ When we say that a random vector lies in a subspace we mean w.p. 1.
}

whitening $\boldsymbol{a}$ on the subspace $\mathcal{V}$ in which it lies, which is referred to as subspace whitening [4], [3]. The noise component in the output of the subspace transformation $x$ is said to be white on $\mathcal{V}$ if its covariance matrix $\boldsymbol{C}_{x}$ is given by

$$
\boldsymbol{C}_{x}=\sigma^{2} P_{\mathcal{V}}=\sigma^{2} \boldsymbol{R} \tilde{\boldsymbol{I}} \boldsymbol{R}^{*}
$$

where the first $K$ columns of $\boldsymbol{R}$ form an orthonormal basis for $\mathcal{V}$ and $K$ is the dimension of $\mathcal{V}$, and $\tilde{\boldsymbol{I}}$ is the $M \times M$ matrix

$$
\tilde{\boldsymbol{I}}=\left[\begin{array}{cc}
\boldsymbol{I}_{K} & 0 \\
0 & 0
\end{array}\right] \text {. }
$$

The condition (6) implies that the representation of the noise component of $\boldsymbol{x}$ in terms of any orthonormal basis for $\mathcal{V}$ is white in the conventional sense. As in the linearly independent case, we seek a subspace whitening transformation $\boldsymbol{W}$ satisfying

$$
\boldsymbol{W}_{a} \boldsymbol{W}^{*}=\sigma^{2} \boldsymbol{W}\left(\boldsymbol{S}^{*} \boldsymbol{S}\right)^{\dagger} \boldsymbol{W}^{*}=\sigma^{2} P_{\mathcal{V}}
$$

such that (5) is minimized. A more elaborate discussion on subspace whitening can be found in [4]. In the remainder of the paper, we use the term whitening to denote both whitening and subspace whitening.

The solution to the general MMSE whitening problem has been obtained in [4], and is incorporated in the following theorem.

Theorem 1 (MMSE Whitening [4]): Let $\boldsymbol{z} \in \mathbb{C}^{M}$ be a zero-mean random vector with covariance matrix $\boldsymbol{C}_{z}=U \boldsymbol{U} \boldsymbol{D} \boldsymbol{U}^{*}$ with $\operatorname{rank}\left(\boldsymbol{C}_{z}\right)=K \leq M$, where $\boldsymbol{U}$ is a unitary matrix and $D$ is a diagonal matrix with the first $K$ elements positive and the remaining diagonal elements equal to 0 . Let $\mathcal{V}$ denote the range space of $\boldsymbol{C}_{z}$ spanned by the first $K$ columns of $\boldsymbol{U}$. Let $\hat{W}$ be any whitening transformation that minimizes the total MSE between the input $\boldsymbol{z}$ and the output $\boldsymbol{h}$ with covariance $\boldsymbol{C}_{h}=c^{2} P_{\mathcal{V}}=c^{2} \boldsymbol{U} \tilde{\boldsymbol{I}} \boldsymbol{U}^{*}$, where $\tilde{\boldsymbol{I}}$ is given by (7) and $c>0$. Then

1) if $K=M$, then $\hat{\boldsymbol{W}}$ is unique and is given by

$$
\hat{\boldsymbol{W}}=c \boldsymbol{U} \boldsymbol{D}^{-1 / 2} \boldsymbol{U}^{*}=c \boldsymbol{C}_{z}^{-1 / 2} ;
$$

2) if $K<M$, then

a) $\hat{W}$ is not unique,

b) $\hat{\boldsymbol{W}}=c \boldsymbol{U}\left(\boldsymbol{D}^{1 / 2}\right)^{\dagger} \boldsymbol{U}^{*}=c\left(\boldsymbol{C}_{z}^{1 / 2}\right)^{\dagger}$ is an optimal whitening transformation.

Furthermore, let $\boldsymbol{W}^{\mathcal{V}}=\hat{\boldsymbol{W}} P_{\mathcal{V}}$ where $P_{\mathcal{V}}$ is an orthogonal projection onto $\mathcal{V}$ and $\hat{\boldsymbol{W}}$ is any optimal whitening transformation. Then

1) $W^{\mathcal{V}}$ is unique, and is given by

$$
\boldsymbol{W}^{\mathcal{V}}=c \boldsymbol{U}\left(\boldsymbol{D}^{1 / 2}\right)^{\dagger} \boldsymbol{U}^{*}=c\left(\boldsymbol{C}_{z}^{1 / 2}\right)^{\dagger}
$$

2) $\hat{W} \boldsymbol{z}=\boldsymbol{W}^{\mathcal{V}} \boldsymbol{z}$ w.p. 1 .

In Fig. 2, the noise component in the input $\boldsymbol{a}$ to the whitening transformation has zero mean and covariance $\boldsymbol{C}_{a}=\sigma^{2}\left(\boldsymbol{S}^{*} \boldsymbol{S}\right)^{\dagger}$. From Theorem 1, an optimal whitening transformation is

$$
\hat{\boldsymbol{W}}=\left(\boldsymbol{S}^{*} \boldsymbol{S}\right)^{1 / 2} .
$$

Note that in Fig. 2, $\boldsymbol{a}$ is restricted to the range space $\mathcal{V}$ of $\boldsymbol{C}_{a}$, since $\mathcal{V}$ is also the range of $\boldsymbol{V}^{*}$ and $\boldsymbol{a}=\boldsymbol{V}^{*} \boldsymbol{r}$. Consequently, the output $\boldsymbol{x}$ is not affected by the action of $\hat{\boldsymbol{W}}$ on $\mathcal{V}^{\perp}$, so that 
any MMSE whitening transformation will result in the same output $\boldsymbol{x}$.

\section{B. Correlation Demodulator Representation of the OMU Demodulator}

As we have seen in Section II, any demodulator of the form of Fig. 2 is equivalent to a correlation demodulator of the form of Fig. 1 with correlating vectors $\boldsymbol{q}_{m}$ that are the columns of $\boldsymbol{Q}=$ $\boldsymbol{V} \boldsymbol{B}^{*}$. Since the OMU demodulator consists of a decorrelator followed by the MMSE whitening transformation $\hat{W}$ given by (9), it is equivalent to a bank of correlators with vectors $\boldsymbol{q}_{m}=$ $\hat{\boldsymbol{g}}_{m}$, where $\hat{\boldsymbol{g}}_{m}=[\hat{\boldsymbol{G}}]_{m}$ and

$$
\hat{\boldsymbol{G}}=\boldsymbol{V} \hat{\boldsymbol{W}}^{*}=\boldsymbol{S}\left(\boldsymbol{S}^{*} \boldsymbol{S}\right)^{\dagger}\left(\boldsymbol{S}^{*} \boldsymbol{S}\right)^{1 / 2}=\boldsymbol{S}\left(\left(\boldsymbol{S}^{*} \boldsymbol{S}\right)^{1 / 2}\right)^{\dagger} .
$$

We now try to gain some insight into the vectors $\hat{\boldsymbol{g}}_{m}$, which we refer to as the OMU vectors.

From (10) and (8) we have that

$$
\hat{\boldsymbol{G}}^{*} \hat{\boldsymbol{G}}=\hat{\boldsymbol{W}} \boldsymbol{V}^{*} \boldsymbol{V} \hat{\boldsymbol{W}}^{*}=\hat{\boldsymbol{W}}\left(\boldsymbol{S}^{*} \boldsymbol{S}\right)^{\dagger} \hat{\boldsymbol{W}}^{*}=P_{\mathcal{V}} .
$$

If the vectors $\boldsymbol{s}_{m}$ are linearly independent, then $P_{\mathcal{V}}=\boldsymbol{I}_{M}$ and from (11) it follows that the vectors $\left\{\hat{\boldsymbol{g}}_{m}, 1 \leq m \leq M\right\}$ are orthonormal. If the vectors $\boldsymbol{s}_{m}$ are linearly dependent, then the vectors $\hat{\boldsymbol{g}}_{m}$ are clearly not orthonormal. Rather, they form a normalized tight frame [20]-[22] for $\mathcal{U}$.

A set of vectors $\left\{\boldsymbol{z}_{m} \in \mathcal{W}, 1 \leq m \leq M\right\}$ form a normalized tight frame for a subspace $\mathcal{W}$ if for any $\boldsymbol{y} \in \mathcal{W}$

$$
\sum_{m=1}^{M}\left|\boldsymbol{z}_{m}^{*} \boldsymbol{y}\right|^{2}=\boldsymbol{y}^{*} \boldsymbol{y}
$$

The condition (12) is equivalent to

$$
\boldsymbol{Z}^{*} \boldsymbol{Z}=P_{\mathcal{N}(\boldsymbol{Z})^{\perp}}
$$

where $\boldsymbol{Z}$ is the matrix of columns $\boldsymbol{z}_{m}$. Alternatively, a set of vectors forms a normalized tight frame for $\mathcal{W}$ if and only if the vectors can be expressed as the orthogonal projections onto $\mathcal{W}$ of a set of orthonormal vectors in a larger space containing $\mathcal{W}$ [22]. Clearly, if $\mathcal{N}(\boldsymbol{Z})^{\perp}=\boldsymbol{I}_{M}$, then (13) implies that the vectors $\boldsymbol{z}_{m}$ are orthonormal.

Comparing (11) with (13) we conclude that a decorrelator demodulator followed by a whitening transformation is equivalent to a bank of correlators with vectors that form a normalized tight frame for $\mathcal{U}$. In the case in which the signature vectors are linearly independent, the normalized tight frame reduces to a set of orthonormal vectors.

Since the MMSE whitening transformation is optimal in some sense, we expect the OMU vectors it defines to also have some form of optimality. The following theorem, proved in the Appendix, asserts that the OMU vectors form the closest orthonormal vectors in the linearly independent case, and the closest normalized tight frame vectors in the linearly dependent case, in a least squares sense to both the decorrelator vectors $\boldsymbol{v}_{m}$ and the signature vectors $\boldsymbol{s}_{m}$.

Theorem 2: Let $\left\{\boldsymbol{s}_{m}, 1 \leq m \leq M\right\}$ denote a set of $M$ signature vectors and let $\left\{\hat{\boldsymbol{g}}_{m}, 1 \leq m \leq M\right\}$ denote the OMU

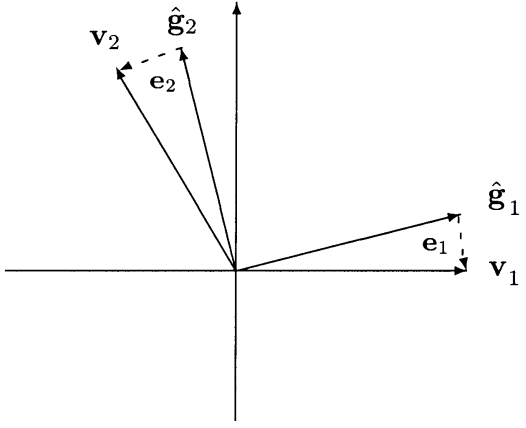

Fig. 3. Two-dimensional example of the OMU vectors. Since the vectors $\boldsymbol{v}_{1}$ and $\boldsymbol{v}_{2}$ are linearly independent, the OMU vectors $\hat{\boldsymbol{g}}_{1}$ and $\hat{\boldsymbol{g}}_{2}$ are chosen to be orthonormal and to minimize $\sum_{i}\left\langle\boldsymbol{e}_{i}, \boldsymbol{e}_{i}\right\rangle=\sum_{i}\left\langle\boldsymbol{v}_{i}-\boldsymbol{g}_{i}, \boldsymbol{v}_{i}-\boldsymbol{g}_{i}\right\rangle$.

vectors that are the correlating vectors of the OMU demodulator. Let

$$
\varepsilon_{\mathrm{LS}}\left(\left\{\boldsymbol{y}_{m}\right\},\left\{\boldsymbol{g}_{m}\right\}\right)=\sum_{m=1}^{M}\left(\boldsymbol{y}_{m}-\boldsymbol{g}_{m}\right)^{*}\left(\boldsymbol{y}_{m}-\boldsymbol{g}_{m}\right)
$$

denote the least squares error between the vectors

$$
\left\{\boldsymbol{y}_{m}, 1 \leq m \leq M\right\} \quad \text { and } \quad\left\{\boldsymbol{g}_{m}, 1 \leq m \leq M\right\} .
$$

Then

1) the vectors

$$
\left\{\hat{\boldsymbol{g}}_{m}, 1 \leq m \leq M\right\}
$$

minimize $\varepsilon_{\mathrm{LS}}\left(\left\{\boldsymbol{v}_{m}\right\},\left\{\boldsymbol{g}_{m}\right\}\right)$ over all vectors $\left\{\boldsymbol{g}_{m}\right\}$ that form a normalized tight frame for $\mathcal{U}$, where $\left\{\boldsymbol{v}_{m}\right\}$ are the decorrelator vectors;

2) the vectors

$$
\left\{\hat{\boldsymbol{g}}_{m}, 1 \leq m \leq M\right\}
$$

minimize $\varepsilon_{\mathrm{LS}}\left(\left\{\boldsymbol{s}_{m}\right\},\left\{\boldsymbol{g}_{m}\right\}\right)$ over all vectors $\left\{\boldsymbol{g}_{m}\right\}$ that form a normalized tight frame for $\mathcal{U}$.

From Theorem 2, it follows that seeking a whitening transformation to minimize the MSE defined by (5) is equivalent to seeking a set of vectors $\boldsymbol{g}_{m}$ that form a normalized tight frame for $\mathcal{U}$, and are closest to the decorrelator vectors $\boldsymbol{v}_{m}$ and the signature vectors $\boldsymbol{s}_{m}$ in the least squares sense. In particular, when the signature vectors are linearly independent, we may interpret the OMU demodulator as a bank of correlators matched to a set of orthonormal vectors that are closest in the least squares sense to the decorrelator vectors and to the signature vectors, as illustrated in Fig. 3. When the signature vectors are linearly dependent, the OMU demodulator can be interpreted as a bank of correlators matched to a set of vectors that are the projections onto $\mathcal{U}$ of a set of orthonormal vectors that are closest in the least squares sense to the decorrelator vectors and to the signature vectors. This problem has been solved in the context of quantum detection [10], general inner product shaping [23], and optimal frame design [22]; the solution in [10], [23], [22] is equal to the OMU vectors given by (10).

\section{MF Representation of the OMU Demodulator}

Let us now consider an MF demodulator followed by an MMSE whitening transformation $\hat{W}^{\mathrm{mf}}$ that minimizes the 
MSE between the vector output $\tilde{\boldsymbol{a}}$ of the MF demodulator and the output $x$ of the whitening transformation, analogous to (5). Since the covariance of the noise component in $\tilde{\boldsymbol{a}}$ is given by $\boldsymbol{S}^{*} \boldsymbol{S}$, from Theorem 1 it follows that $\hat{\boldsymbol{W}}^{\mathrm{mf}}=\left(\left(\boldsymbol{S}^{*} \boldsymbol{S}\right)^{1 / 2}\right)^{\dagger}$. Therefore, the vector output $x$ of an MF demodulator followed by an MMSE whitening transformation is given by

$$
\boldsymbol{x}=\hat{\boldsymbol{W}}^{\mathrm{mf}} \tilde{\boldsymbol{a}}=\left(\left(\boldsymbol{S}^{*} \boldsymbol{S}\right)^{1 / 2}\right)^{\dagger} \boldsymbol{S}^{*} \boldsymbol{r}
$$

Alternatively, using the decorrelator as a front end, the output of Fig. 2 with $B=\hat{W}$ given by (9) is

$$
\begin{aligned}
\boldsymbol{x} & =\hat{\boldsymbol{W}} \boldsymbol{a}=\hat{\boldsymbol{W}} \boldsymbol{V}^{*} \boldsymbol{r} \\
& =\left(\boldsymbol{S}^{*} \boldsymbol{S}\right)^{1 / 2}\left(\boldsymbol{S}^{*} \boldsymbol{S}\right)^{\dagger} \boldsymbol{S}^{*} \boldsymbol{r}=\left(\left(\boldsymbol{S}^{*} \boldsymbol{S}\right)^{1 / 2}\right)^{\dagger} \boldsymbol{S}^{*} \boldsymbol{r} .
\end{aligned}
$$

Comparing (15) with (16) we conclude that the two outputs are equal. Note also that (15) and (16) are consistent with (10).

\section{Computational Aspects}

To implement the OMU demodulator efficiently, we may use the form of Fig. 1 where the vectors $\boldsymbol{q}_{m}=\hat{\boldsymbol{g}}_{m}$, and $\hat{\boldsymbol{g}}_{m}$ are the OMU vectors. The OMU vectors may be computed efficiently by expressing $\hat{\boldsymbol{G}}$ either in terms of the polar decomposition [19] or the singular value decomposition (SVD) of $\boldsymbol{S}$.

First, we show that $\hat{G}$ is the orthogonal projection onto the space $\mathcal{U}$ spanned by the vectors $\boldsymbol{s}_{m}$ of the partial isometry in a polar decomposition of $\boldsymbol{S}$ [23].

Let $\boldsymbol{A}$ denote an arbitrary $N \times M$ matrix with ${ }^{3} N \geq M$. Then, $\boldsymbol{A}$ has a polar decomposition [24], [19]

$$
A=H P
$$

where $\boldsymbol{H}$ is an $N \times M$ partial isometry that satisfies $\boldsymbol{H}^{*} \boldsymbol{H}=\boldsymbol{I}_{N}$, and $\boldsymbol{P}=\left(\boldsymbol{A}^{*} \boldsymbol{A}\right)^{1 / 2}$. The Hermitian factor $\boldsymbol{P}$ is always unique; the partial isometry $\boldsymbol{H}$ is unique if and only if $\boldsymbol{A}$ has full column rank. When $\boldsymbol{A}$ has full column rank, the columns of the partial isometry $\boldsymbol{H}$ are the orthonormal vectors that are closest in a least squares sense to the columns of $\boldsymbol{A}$ [24], [23]. If $\boldsymbol{A}$ does not have full rank, then there are many possible choices for $\boldsymbol{H}$. However, for any such choice, the columns of $P_{\mathcal{R}(\boldsymbol{A})} H$, where $P_{\mathcal{R}(\boldsymbol{A})}$ is an orthogonal projection onto $\mathcal{R}(\boldsymbol{A})$, form a normalized tight frame for $\mathcal{R}(\boldsymbol{A})$ that is closest in a least squares sense to the columns of $\boldsymbol{A}$.

When the signatures $\boldsymbol{s}_{m}$ are linearly independent, it follows from Theorem 3 that the OMU vectors are the orthonormal vectors that are closest in a least squares to the signature vectors $\boldsymbol{s}_{m}$, and when the signature vectors are linearly dependent, the OMU vectors are the normalized tight frame vectors that are closest in a least squares to the signature vectors $\boldsymbol{s}_{m}$. From the properties of the polar decomposition, we conclude that in the linearly independent case the OMU vectors are the columns of the partial isometry in the polar decomposition of $\boldsymbol{S}$. In the linearly dependent case, the OMU vectors are the columns of the projection onto $\mathcal{U}$ of any partial isometry in a polar decomposition of $\boldsymbol{S}$.

Alternatively, we can also express $\hat{G}$ in terms of the SVD of $\boldsymbol{S}$. Let the SVD of $\boldsymbol{S}$ be given by $\boldsymbol{S}=\boldsymbol{U} \boldsymbol{\Sigma} \boldsymbol{R}^{*}$, where $\boldsymbol{U}$ and $\boldsymbol{R}$

${ }^{3}$ If $M>N$, then we can define the polar decomposition for $\boldsymbol{A}^{*}$. are unitary matrices and $\Sigma$ is a diagonal $N \times M$ matrix. Then we may express $\hat{G}$ as

$$
\hat{\boldsymbol{G}}=\boldsymbol{U} \tilde{\boldsymbol{I}}_{K} \boldsymbol{R}^{*}
$$

where $\tilde{\boldsymbol{I}}_{K}$ is the $N \times M$ matrix given by

$$
\tilde{I}_{K}=\left[\begin{array}{cc}
I_{K} & 0 \\
0 & 0
\end{array}\right] .
$$

Since the OMU vectors can be expressed in terms of the polar decomposition and the SVD of $\boldsymbol{S}$, they may be computed very efficiently exploiting the many known efficient algorithms for computing either the polar decomposition or the SVD (see, e.g., [15], [25], [24]).

\section{A. Summary of the OMU Representation}

We summarize our results regarding the OMU demodulator in the following theorem.

Theorem 3 (OMU Demodulator): Let $\left\{\boldsymbol{s}_{m}, 1 \leq m \leq M\right\}$ denote a set of $M$ signature vectors that span a $K$-dimensional subspace $\mathcal{U} \subseteq \mathbb{C}^{M}$, where $K \leq M$. Let $\left\{\hat{\boldsymbol{g}}_{m}, 1 \leq m \leq M\right\}$ denote the OMU vectors that are the correlating vectors of the OMU demodulator. Let $\boldsymbol{S}$ and $\hat{\boldsymbol{G}}$ denote the matrices of columns $\boldsymbol{s}_{m}$ and $\hat{\boldsymbol{g}}_{m}$, respectively, and let $\boldsymbol{S}$ have an SVD $\boldsymbol{S}=\boldsymbol{U} \boldsymbol{\Sigma} \boldsymbol{R}^{*}$. Then

$$
\hat{\boldsymbol{G}}=\boldsymbol{S}\left(\left(\boldsymbol{S}^{*} \boldsymbol{S}\right)^{1 / 2}\right)^{\dagger}=\boldsymbol{U} \tilde{\boldsymbol{I}}_{K} \boldsymbol{R}^{*}
$$

where $\tilde{I}_{K}$ is given by (19), and is equal to the orthogonal projection onto $\mathcal{U}$ of the partial isometry in a polar decomposition of $\boldsymbol{S}$. In addition,

1) the OMU demodulator can be realized by a decorrelator demodulator followed by an MMSE whitening transformation $\hat{W}=\left(\boldsymbol{S}^{*} \boldsymbol{S}\right)^{1 / 2}$

2) the OMU demodulator can be realized by an MF demodulator followed by an MMSE whitening transformation $\hat{\boldsymbol{W}}^{\mathrm{mf}}=\left(\left(\boldsymbol{S}^{*} \boldsymbol{S}\right)^{1 / 2}\right)^{\dagger}$

3) the vectors $\left\{\hat{\boldsymbol{g}}_{m}\right\}$ minimize the least squares error $\varepsilon_{\mathrm{LS}}\left(\left\{\boldsymbol{v}_{m}\right\},\left\{\boldsymbol{g}_{m}\right\}\right)$ given by (14), i.e., they form the closest normalized tight frame to the decorrelator vectors $\left\{\boldsymbol{v}_{m}\right\}$

4) the vectors $\left\{\hat{\boldsymbol{g}}_{m}\right\}$ minimize the least squares error $\varepsilon_{\mathrm{LS}}\left(\left\{\boldsymbol{s}_{m}\right\},\left\{\boldsymbol{g}_{m}\right\}\right)$, i.e., they form the closest normalized tight frame to the signature vectors $\left\{\boldsymbol{s}_{m}\right\}$.

\section{FuRTher OPTIMALITY PROPERITES OF THE OMU DEMODULATOR}

To derive the OMU demodulator, we proposed whitening the output of the decorrelator demodulator and then choosing the whitening transformation to minimize the MSE between the noise components in the input and output of the whitening transformation. In this section, we show that this choice of whitening transformation has the additional property that among all possible whitening transformations, it minimizes the total MAI and maximizes the total SNR in the output of the transformation for signature vectors that have a strong 
symmetry property. Furthermore, for nearly orthogonal signature vectors [26], the MMSE whitening transformation approximately minimizes the total MAI and approximately maximizes the total SNR.

For simplicity of exposition, we assume throughout this section that $E\left(A_{m}^{2}\right)=1$ for all $m$; the results extend in a straightforward way to the general case in which the powers $E\left(A_{m}^{2}\right)$ are not equal.

\section{A. Minimum Total MAI Whitening Transformation}

We have seen that (in the linearly independent case) the decorrelator eliminates the MAI by inverting the multiuser channel, but in the process may enhance the white noise. We proposed compensating for this possible noise enhancement by whitening the noise component in the output of the decorrelator prior to detection. However, the whitening transformation introduces some MAI into the outputs $x_{m}$. Indeed, the data component in the output $\boldsymbol{x}$ of the whitening transformation is the vector $\boldsymbol{W} \boldsymbol{A b}$, whose $m$ th component is

$$
\sum_{k=1}^{M}[\boldsymbol{W}]_{m k} A_{k} b_{k}=x_{m}^{S}+x_{m}^{I}
$$

where $x_{m}^{S}=[\boldsymbol{W}]_{m m} A_{m} b_{m}$ is the signal component in $x_{m}$, and

$$
x_{m}^{I}=\sum_{k=1, k \neq m}^{M}[\boldsymbol{W}]_{m k} A_{k} b_{k}
$$

is the MAI component in $x_{m}$. We may, therefore, choose $\boldsymbol{W}$ to minimize the total MAI in the output $\boldsymbol{x}$, or equivalently, to maximize the total SIR in $\boldsymbol{x}$ given by

$$
\begin{aligned}
\operatorname{SIR}_{T} & =\frac{\sum_{m=1}^{M} E\left(\left(x_{m}^{S}\right)^{2}\right)}{\sum_{m=1}^{M} E\left(\left(x_{m}^{I}\right)^{2}\right)} \\
& =\frac{\sum_{m=1}^{M}\left|[W]_{m m}\right|^{2}}{\sum_{m=1}^{M} \sum_{k=1, k \neq m}^{M}\left|[\boldsymbol{W}]_{m k}\right|^{2}}
\end{aligned}
$$

subject to the whitening constraint

$$
\boldsymbol{W} \boldsymbol{C}_{a} \boldsymbol{W}^{*}=\sigma^{2} \boldsymbol{I}_{M}
$$

or the subspace whitening constraint

$$
\boldsymbol{W} \boldsymbol{C}_{a} \boldsymbol{W}^{*}=\sigma^{2} P_{\mathcal{V}}
$$

where $C_{a}$ is the covariance of the noise component in $\boldsymbol{a}$ given by (3), and $\mathcal{V}=\mathcal{N}\left(\boldsymbol{C}_{a}\right)^{\perp}$.

We first consider the case of linearly independent signature vectors. In this case, we can simplify the expression for $\operatorname{SIR}_{T}$ given by (21) as follows. Since $\boldsymbol{W}$ must be invertible, (22) reduces to

$$
\boldsymbol{W}^{*} \boldsymbol{W}=\sigma^{2} \boldsymbol{C}_{a}^{-1}
$$

From (24) we conclude that

$$
\alpha \triangleq \operatorname{tr}\left(\boldsymbol{W}^{*} \boldsymbol{W}\right)=\sum_{m, k=1}^{M}\left|[\boldsymbol{W}]_{m k}\right|^{2}
$$

is constant, independent of the choice of $\boldsymbol{W}$. Expressing (21) as

$$
\operatorname{SIR}_{T}=\frac{\sum_{m=1}^{M}\left|[\boldsymbol{W}]_{m m}\right|^{2}}{\alpha-\sum_{m=1}^{M}\left|[\boldsymbol{W}]_{m m}\right|^{2}},
$$

it follows that maximizing $\mathrm{SIR}_{T}$ subject to (22) is equivalent to maximizing

$$
\Gamma=\sum_{m=1}^{M}\left|[\boldsymbol{W}]_{m m}\right|^{2}
$$

subject to (22).

Let $\boldsymbol{C}_{a}$ have an eigendecomposition $\boldsymbol{C}_{a}=\boldsymbol{R} \boldsymbol{\Lambda} \boldsymbol{R}^{*}$, where $\boldsymbol{\Lambda}$ is a diagonal matrix with diagonal elements $\lambda_{m}>0$. Then, from (24), and using the properties of the SVD, we have that

$$
\boldsymbol{W}=\sigma \boldsymbol{Z}^{*} \boldsymbol{\Lambda}^{-1 / 2} \boldsymbol{R}^{*}
$$

for some unitary matrix $\boldsymbol{Z}^{*}$. Let $\boldsymbol{z}_{m}$ denote the columns of $\boldsymbol{Z}$, and let $\boldsymbol{y}_{m}$ denote the columns of $\boldsymbol{Y}=\sigma \boldsymbol{\Lambda}^{-1 / 2} \boldsymbol{R}^{*}$. Then $[\boldsymbol{W}]_{m m}=\boldsymbol{z}_{m}^{*} \boldsymbol{y}_{m}$, and

$$
\Gamma=\sum_{m=1}^{M}\left|[W]_{m m}\right|^{2}=\sum_{m=1}^{M}\left|\boldsymbol{z}_{m}^{*} \boldsymbol{y}_{m}\right|^{2} .
$$

Thus, the problem of maximizing (21) subject to (22) reduces to seeking a set of orthonormal vectors $\boldsymbol{z}_{m}$ that maximize (28).

When the signature vectors are linearly dependent, we can show that the design problem of (21) and (23) reduces to seeking a set of vectors $\boldsymbol{z}_{m}$ that form a normalized tight frame and maximize (28).

This is a reasonable approach to choosing the whitening transformation $W$ and may even seem more intuitive then minimizing the MSE given by (5). However, unlike the latter problem, this problem is hard to solve analytically. Nonetheless, we now show that we can formulate this problem within the framework of quantum detection, and then rely on results obtained in that context.

In a quantum detection problem, a system is prepared in one of $M$ known (pure) states that are described by vectors $\boldsymbol{s}_{m}$ in an abstract Hilbert space $\mathcal{H}$, and the problem is to detect the state prepared by performing a measurement on the system. The measurement is described in terms of a set of measurement vectors $\boldsymbol{q}_{m}$ which are orthonormal if the states $\boldsymbol{s}_{m}$ are linearly independent, or which form a normalized tight frame if the states are linearly dependent. ${ }^{4}$ Given a set of measurement vectors $\boldsymbol{q}_{m}$,

\footnotetext{
${ }^{4}$ In a quantum context, a normalized tight frame is referred to as a rank-one positive operator-valued measure (POVM).
} 
and assuming equal prior probabilities on the different states, the probability of correct detection is given by [27]

$$
P_{q d}=\frac{1}{M} \sum_{m=1}^{M}\left|\left\langle\boldsymbol{q}_{m}, \boldsymbol{s}_{m}\right\rangle\right|^{2}
$$

where $\left\langle\boldsymbol{q}_{m}, \boldsymbol{s}_{m}\right\rangle$ denotes the inner product between the vectors $\boldsymbol{q}_{m}$ and $\boldsymbol{s}_{m}$. Comparing (28) with (29), we see that choosing the measurement vectors to maximize the probability of correct detection subject to an orthogonality or normalized tight frame constraint is equivalent to choosing a whitening transformation to maximize $\mathrm{SIR}_{T}$ subject to the corresponding constraint. We may, therefore, interpret the design problem of (28) as a quantum detection problem, and then apply results derived in that context.

Based on results derived in a quantum detection context [10], [28], it can be shown that when the signature vectors have a strong symmetry property called geometric uniformity [9], the vectors $\boldsymbol{z}_{m}$ maximizing (28) are equal to the columns of the unitary matrix $\boldsymbol{R}^{*}$ in the eigendecomposition of $\boldsymbol{C}_{a}$. From (27), it then follows that the whitening transformation that maximizes $\mathrm{SIR}_{T}$ is given by $\boldsymbol{W}=\boldsymbol{R}\left(\boldsymbol{\Lambda}^{1 / 2}\right)^{\dagger} \boldsymbol{R}^{*}=\sigma\left(\boldsymbol{C}_{a}^{1 / 2}\right)^{\dagger}=\hat{\boldsymbol{W}}$, where $\hat{W}$ is the MMSE whitening transformation given by (9).

A set of vectors is geometrically uniform (GU) if given any two vectors $\boldsymbol{s}_{k}$ and $\boldsymbol{s}_{m}$ in the set, there is an isometry (a normpreserving linear transformation) $\boldsymbol{T}_{k m}$ that transforms $\boldsymbol{s}_{k}$ into $\boldsymbol{s}_{m}$ while leaving the rest of the set invariant [9], so that $\boldsymbol{T}_{k m}$ permutes the set. Thus, for every $k, \boldsymbol{T}_{k m} \boldsymbol{s}_{k}=\boldsymbol{s}_{m}$ for some $m$. Intuitively, a vector set is GU if it "looks the same" from any of the vectors in the set. It can be shown [23] that a set of vectors with real Gram matrix is GU if and only if the Gram matrix of inner products of the vectors in the set has the property that the second and subsequent rows are permutations of the first row. For example, if the Gram matrix is a circulant matrix, ${ }^{5}$ then the corresponding signature set is GU. In particular, if the inner product between any two distinct vectors in the set is equal to a constant $\rho$, and the norm of all the vectors is the same, then the corresponding Gram matrix is circulant and the vector set is GU. In this case, assuming normalized vectors, the rows of the Gram matrix are permutations of the sequence $\{1, \rho, \rho, \ldots, \rho\}$.

A common choice for signature vectors in a direct-sequence CDMA system are PN sequences corresponding to maximallength shift-register sequences [1], [11]. These sequences have the property that the inner product between any two distinct sequences is equal to a constant. Thus, for this choice of signature vectors, the OMU demodulator maximizes $\mathrm{SIR}_{T}$ subject to the constraint that the outputs of the demodulator are uncorrelated on the space in which they lie.

Further results regarding the whitening or subspace whitening transformation maximizing $\operatorname{SIR}_{T}$ that follow from results pertaining to quantum detection are that if the signature vectors are

\footnotetext{
${ }^{5} \mathrm{~A}$ circulant matrix is a matrix where every row (or column) is obtained by a right circular shift (by one position) of the previous row (or column). An example is
}

$$
\left[\begin{array}{lll}
a_{0} & a_{2} & a_{1} \\
a_{1} & a_{0} & a_{2} \\
a_{2} & a_{1} & a_{0}
\end{array}\right] .
$$

nearly orthogonal, then $\hat{\boldsymbol{W}}$ also approximately maximizes $\operatorname{SIR}_{T}$ [26]. Iterative algorithms [29], [26], [23] can be used to maximize $\mathrm{SIR}_{T}$ when the signature sets are arbitrary.

\section{B. Maximum Total SNR Whitening Transformation}

From results in [30], [3] that also exploit the connection with the quantum detection problem, it can be shown that for GU signature vectors the whitening transformation in the OMU demodulator also maximizes the total output SNR defined by $\mathrm{SNR}_{T}=\sum_{m=1}^{M} \mathrm{SNR}_{m}$, where $\mathrm{SNR}_{m}$ is the SNR at the $m$ th output of the whitening transformation and is defined by $\mathrm{SNR}_{m}=E\left(\left(x_{m}^{S}\right)^{2}\right) / \sigma^{2}$. Therefore, for PN sequences, the OMU demodulator maximizes the total output SNR and minimizes the MAI subject to the decorrelation constraint. Furthermore, for nearly orthogonal signature vectors [26], the MMSE whitening transformation approximately maximizes the total output SNR.

\section{PERformance AnAlysis}

In this section, we discuss the theoretical performance of the OMU receiver. Specifically, in Sections VI-A and VI-B, we derive exact and approximate expressions for the probability of error using the OMU receiver. In Section VI-C, we analyze the large-system performance of the receiver assuming random Gaussian signature vectors and accurate power control. Our analysis indicates that in many cases the OMU receiver can lead to a substantial improvement in performance over the decorrelator and the MF receivers, which motivates the use of this receiver.

\section{A. Exact Probability of Error}

The detector input of the OMU receiver defined via (10) is

$$
\boldsymbol{x}=\left(\left(\boldsymbol{S}^{*} \boldsymbol{S}\right)^{1 / 2}\right)^{\dagger} \boldsymbol{S}^{*} \boldsymbol{r}=\left(\boldsymbol{S}^{*} \boldsymbol{S}\right)^{1 / 2} \boldsymbol{A} \boldsymbol{b}+\left(\left(\boldsymbol{S}^{*} \boldsymbol{S}\right)^{1 / 2}\right)^{\dagger} \boldsymbol{S}^{*} \boldsymbol{n}
$$

Each component of the detector input vector can be decomposed into

$$
x_{m}=x_{m}^{S}+x_{m}^{I}+x_{m}^{N}
$$

where the terms

$$
\begin{aligned}
& x_{m}^{S}=\left[\left(\boldsymbol{S}^{*} \boldsymbol{S}\right)^{1 / 2}\right]_{m m} A_{m} b_{m} \\
& x_{m}^{I}=\sum_{k \neq m}\left[\left(\boldsymbol{S}^{*} \boldsymbol{S}\right)^{1 / 2}\right]_{m k} A_{k} b_{k} \\
& x_{m}^{N}=\left[\left(\left(\boldsymbol{S}^{*} \boldsymbol{S}\right)^{1 / 2}\right)^{\dagger}\right]_{m}^{*} \boldsymbol{S}^{*} \boldsymbol{n}
\end{aligned}
$$

represent the desired signal, the MAI, and the noise, respectively. Conditioned on $\boldsymbol{b}$, the decision statistic $x_{m}$ is Gaussian with mean $x_{m}^{S}+x_{m}^{I}$ and variance

$$
\begin{aligned}
\operatorname{var}\left(x_{m}^{N}\right) & =\sigma^{2}\left[\left(\left(\boldsymbol{S}^{*} \boldsymbol{S}\right)^{1 / 2}\right)^{\dagger}\right]_{m}^{*} \boldsymbol{S}^{*} \boldsymbol{S}\left[\left(\left(\boldsymbol{S}^{*} \boldsymbol{S}\right)^{1 / 2}\right)^{\dagger}\right]_{m} \\
& =\sigma^{2}\left[\left(\left(\boldsymbol{S}^{*} \boldsymbol{S}\right)^{1 / 2}\right)^{\dagger}\right]_{m}^{*}\left[\left(\boldsymbol{S}^{*} \boldsymbol{S}\right)^{1 / 2}\right]_{m} \\
& =\sigma^{2}\left[P_{\mathcal{V}}\right]_{m m}
\end{aligned}
$$




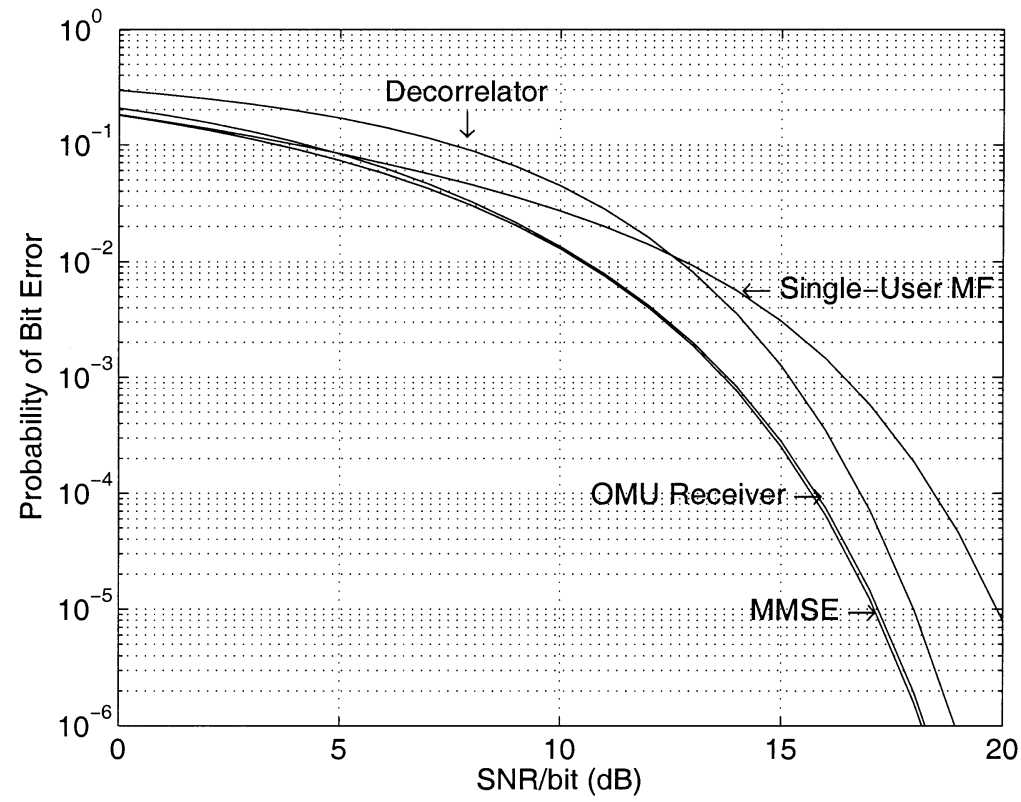

Fig. 4. Probability of bit error with three users and cross correlation $\rho=0.8$. The amplitude $A_{1}$ of the desired user is two times greater than the amplitude $A_{2}$ of the second user and four times greater than the amplitude $A_{3}$ of the third user.

Taking into consideration all possibilities of $\boldsymbol{b}$, the resulting probability of error for the $m$ th user is

$$
\begin{gathered}
P_{m}(\sigma)=\frac{1}{2^{M-1}} \sum_{e_{1} \in\{-1,1\}} \cdots \sum_{\substack{e_{k} \in\{-1,1\} \\
k \neq m}} \cdots \sum_{e_{M} \in\{-1,1\}} \\
. \mathcal{Q}\left(\frac{\left[\left(\boldsymbol{S}^{*} \boldsymbol{S}\right)^{1 / 2}\right]_{m m} A_{m}}{\sigma \sqrt{\left[P_{\mathcal{V}}\right]_{m m}}}+\sum_{k \neq m} \frac{\left[\left(\boldsymbol{S}^{*} \boldsymbol{S}\right)^{1 / 2}\right]_{m k} A_{k}}{\sigma \sqrt{\left[P_{\mathcal{V}}\right]_{m m}}} e_{k}\right)
\end{gathered}
$$

where

$$
\mathcal{Q}(v)=\frac{1}{\sqrt{2 \pi}} \int_{v}^{\infty} e^{-t^{2} / 2} d t .
$$

Fig. 4 evaluates (36) in the case of three users with cross correlation $\rho=0.8$, where the first user, the desired user, has two interferers such that $A_{2} / A_{1}=0.5$ and $A_{3} / A_{1}=0.25$. The corresponding curves for the single-user MF, decorrelator, and linear MMSE receiver are plotted for comparison. The OMU receiver performs similarly to the linear MMSE receiver at all SNR, and better than the decorrelator and the single-user MF.

Next, we examine the scenario in which the desired user has four interferers such that $A_{m} / A_{1}=1$ for $m=2,3,4,5$. In Fig. 5, where the cross correlation of the signatures is shown in (38) at the bottom of the page, the OMU receiver performs significantly better than the decorrelator and the MF. Moreover, the OMU receiver performs slightly better than the linear MMSE receiver at high SNR (although the linear MMSE receiver minimizes the MSE, it does not necessarily minimize the probability of error, especially when the MAI is not approximately Gaussian). In Fig. 6, where the signatures are dependent and the cross correlation is given by the rank-deficient matrix, shown in (39) at the bottom of the page, the OMU receiver again outperforms the decorrelator and the MF.

$$
\boldsymbol{S}^{*} \boldsymbol{S}=\left[\begin{array}{rrrrr}
1.0000 & -0.0650 & 0.0451 & 0.3560 & 0.0629 \\
-0.0650 & 1.0000 & 0.0589 & -0.4206 & 0.0125 \\
0.0451 & 0.0589 & 1.0000 & 0.5864 & -0.0866 \\
0.3560 & -0.4206 & 0.5864 & 1.0000 & 0.5522 \\
0.0629 & 0.0125 & -0.0866 & 0.5522 & 1.0000
\end{array}\right]
$$

$$
\boldsymbol{S}^{*} \boldsymbol{S}=\left[\begin{array}{rrrrr}
1.0000 & -0.1380 & 0.2402 & -0.1894 & -0.4176 \\
-0.1380 & 1.0000 & -0.5466 & -0.1082 & 0.8671 \\
0.2402 & -0.5466 & 1.0000 & 0.8021 & -0.2133 \\
-0.1894 & -0.1082 & 0.8021 & 1.0000 & 0.3531 \\
-0.4176 & 0.8671 & -0.2133 & 0.3531 & 1.0000
\end{array}\right]
$$




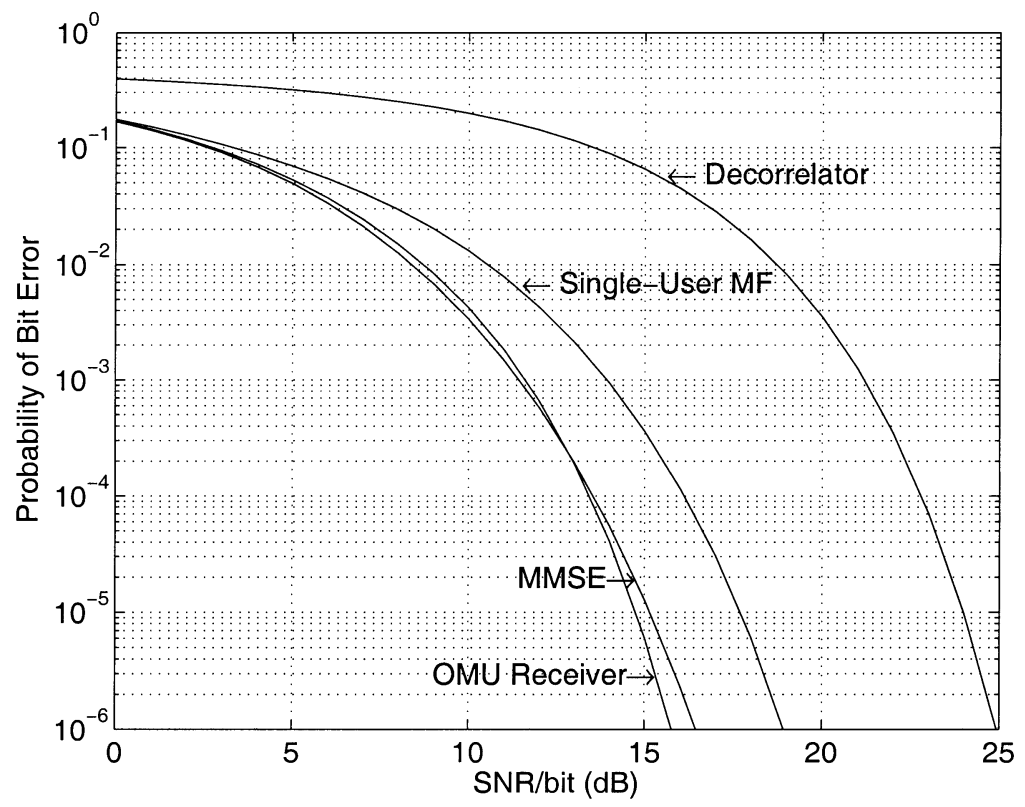

Fig. 5. Probability of bit error with five equal-energy users and linearly independent signatures with cross correlation given by (38).

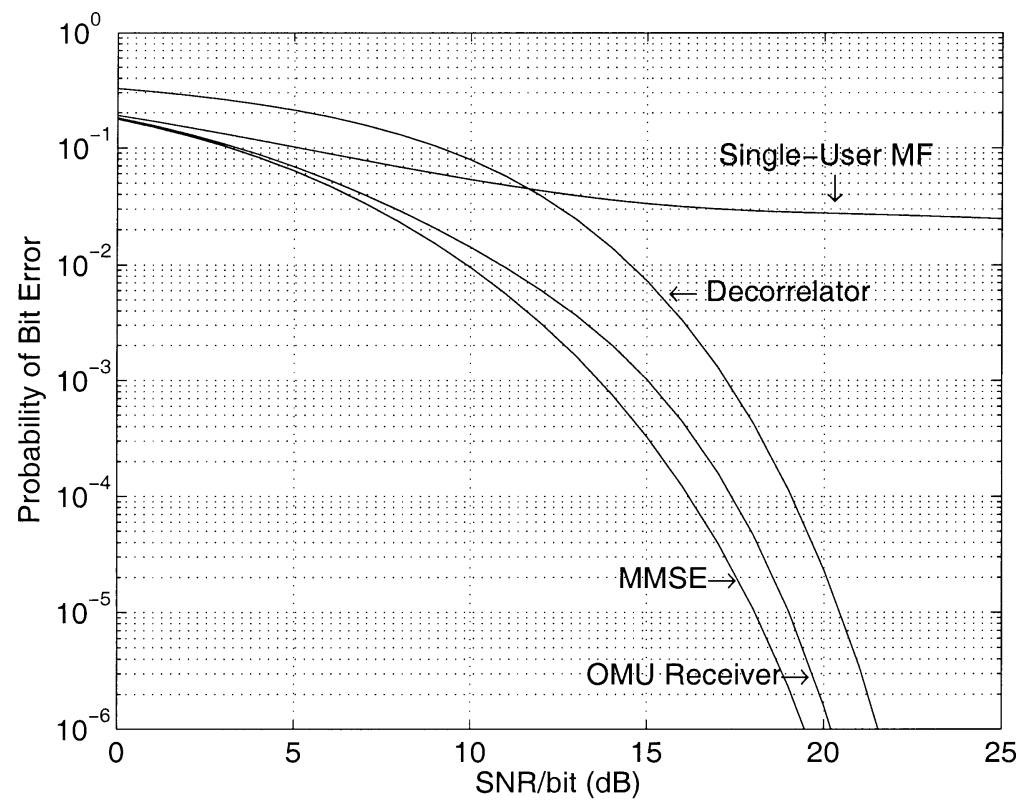

Fig. 6. Probability of bit error with five equal-energy users and linearly dependent signatures with cross correlation given by (39).

B. SINR and Approximating the Probability of Error

From (32)-(34), the terms $x_{m}^{S}, x_{m}^{I}$, and $x_{m}^{N}$ are mutually independent and zero-mean, and have variances

$$
\begin{aligned}
\operatorname{var}\left(x_{m}^{S}\right) & =\left[\left(\boldsymbol{S}^{*} \boldsymbol{S}\right)^{1 / 2}\right]_{m m}^{2} A_{m}^{2} \\
\operatorname{var}\left(x_{m}^{I}\right) & =\left[\left(\boldsymbol{S}^{*} \boldsymbol{S}\right)^{1 / 2}\right]_{m}^{*} \boldsymbol{A}^{2}\left[\left(\boldsymbol{S}^{*} \boldsymbol{S}\right)^{1 / 2}\right]_{m}-\left[\left(\boldsymbol{S}^{*} \boldsymbol{S}\right)^{1 / 2}\right]_{m m}^{2} A_{m}^{2}
\end{aligned}
$$

$$
\operatorname{var}\left(x_{m}^{N}\right)=\sigma^{2}[P \mathcal{V}]_{m m} .
$$

The SINR at the detector for the $m$ th user is therefore shown in (43) at the bottom of the page.

In the case of accurate power control, i.e., $\boldsymbol{A}=A \boldsymbol{I}_{M}$, we can simplify (43) to

$$
\gamma_{m}=\frac{\left[\left(\boldsymbol{S}^{*} \boldsymbol{S}\right)^{1 / 2}\right]_{m m}^{2}}{\zeta\left[P_{\mathcal{V}}\right]_{m m}+\left[\boldsymbol{S}^{*} \boldsymbol{S}\right]_{m m}-\left[\left(\boldsymbol{S}^{*} \boldsymbol{S}\right)^{1 / 2}\right]_{m m}^{2}}
$$

$$
\gamma_{m}=\frac{\left[\left(\boldsymbol{S}^{*} \boldsymbol{S}\right)^{1 / 2}\right]_{m m}^{2} A_{m}^{2}}{\sigma^{2}\left[P_{\mathcal{V}}\right]_{m m}+\left[\left(\boldsymbol{S}^{*} \boldsymbol{S}\right)^{1 / 2}\right]_{m}^{*} \boldsymbol{A}^{2}\left[\left(\boldsymbol{S}^{*} \boldsymbol{S}\right)^{1 / 2}\right]_{m}-\left[\left(\boldsymbol{S}^{*} \boldsymbol{S}\right)^{1 / 2}\right]_{m m}^{2} A_{m}^{2}}
$$




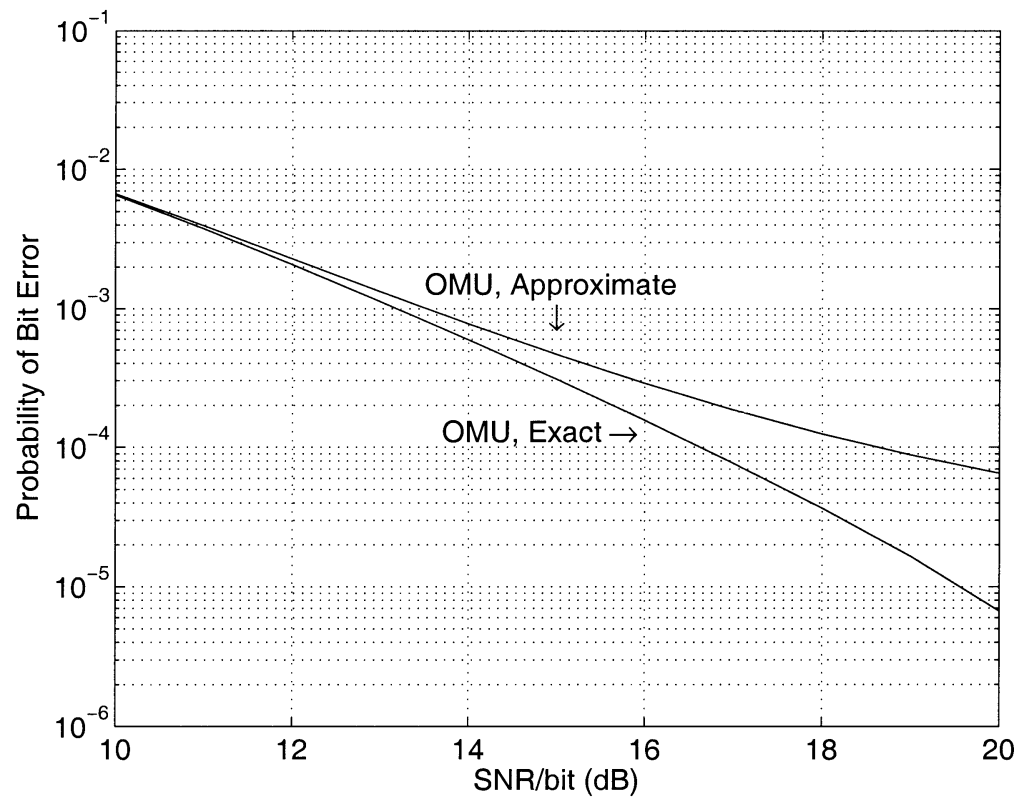

Fig. 7. The exact and Gaussian-approximated probability of bit error for the OMU detector, with 10 equal-energy users and identical cross correlations $\rho=0.2$.

where

$$
\frac{1}{\zeta}=\frac{A^{2}}{\sigma^{2}}
$$

is the received SNR. An alternate form for (44), which will be more convenient for the analysis in Section VI-C, is

$$
\gamma_{m}=\frac{1}{1-\frac{\left[\left(\boldsymbol{S}^{*} \boldsymbol{S}\right)^{1 / 2}\right]_{m m}^{2}}{\zeta\left[\boldsymbol{P}_{\mathcal{V}}\right]_{m m}+\left[\boldsymbol{S}^{*} \boldsymbol{S}\right]_{m m}}}-1
$$

Assuming $x_{m}^{I}+x_{m}^{N}$ is Gaussian, the probability of error can be approximated as

$$
P_{m}(\sigma) \approx \mathcal{Q}\left(\sqrt{\gamma_{m}}\right) .
$$

At low SNR, the Gaussian approximation is acceptable because Gaussian noise is the dominant impairment. However, at high SNR, the discrete distribution of the MAI is poorly approximated by a Gaussian distribution, especially at the tails of the distribution where the bit-error rate is determined. Thus, we do not expect (47) to be particularly accurate at high SNR.

In Fig. 7, we compare the accuracy of the Gaussian approximation in (47) to the exact bit-error rate expression in (36), with 10 equal-energy users and identical cross correlations $\rho=0.2$. As expected, the approximation is useful at low SNR, but becomes less so at high SNR.

\section{Asymptotic Large System Performance}

The following theorem characterizes the performance of the OMU receiver in the large system limit when random Gaussian signatures and accurate power control are used. The method we use in its proof can be easily modified to characterize the performance of other multiuser detectors in the large system limit as well. For example, the method can be used to derive the asymptotic SINR for the MF detector, and it has been recently used to derive the asymptotic SINR for the decorrelator [12].

Theorem 4: Let the elements of the $N \times M$ signature matrix $\boldsymbol{S}$ be independent $\mathcal{C N}(0,1 / N)$, and let the matrix of amplitudes
$\boldsymbol{A}$ be expressible as $A \boldsymbol{I}_{M}$. Then, in the limit as $M \rightarrow \infty$ with $\beta \triangleq M / N$ held constant, the SINR for each user at the OMU demodulator output satisfies ${ }^{6}$

$$
\gamma_{m} \stackrel{\text { m.s. }}{\longrightarrow} \frac{1}{1-\frac{\eta_{2}\left[\left(\eta_{1}+\eta_{2}\right) E\left(\sqrt{1-\eta_{1} / \eta_{2}}\right)-2 \eta_{1} K\left(\sqrt{1-\eta_{1} / \eta_{2}}\right)\right]^{2}}{9 \pi^{2} \beta^{2}(\zeta+1)}}-1
$$

when the signature vectors are linearly independent, and

$$
\gamma_{m} \stackrel{\text { m.s. }}{\longrightarrow} \frac{1}{1-\frac{\eta_{2}\left[\left(\eta_{1}+\eta_{2}\right) E\left(\sqrt{1-\eta_{1} / \eta_{2}}\right)-2 \eta_{1} K\left(\sqrt{1-\eta_{1} / \eta_{2}}\right)\right]^{2}}{9 \pi^{2} \beta^{2}(\zeta / \beta+1)}}-1
$$

when the signature vectors are linearly dependent, where [32]

$$
\begin{aligned}
K(k) & =\int_{0}^{\pi / 2} \frac{d t}{\sqrt{1-k^{2} \sin ^{2} t}} \\
& =\int_{0}^{1} \frac{d x}{\sqrt{\left(1-x^{2}\right)\left(1-k^{2} x^{2}\right)}} \\
E(k) & =\int_{0}^{\pi / 2} \sqrt{1-k^{2} \sin ^{2} t} d t \\
& =\int_{0}^{1} \sqrt{\frac{1-k^{2} x^{2}}{1-x^{2}}} d x
\end{aligned}
$$

are the complete elliptic integrals of the first and second kinds, respectively, and

$$
\begin{aligned}
& \eta_{1}=(1-\sqrt{\beta})^{2} \\
& \eta_{2}=(1+\sqrt{\beta})^{2} .
\end{aligned}
$$

Proof: We begin by presenting the following lemma [12] on Wishart matrices, ${ }^{7}$ which have the form $\boldsymbol{S}^{*} \boldsymbol{S}$ with the ele-

\footnotetext{
${ }^{6}$ We use the notation $\stackrel{\mathrm{m} . \mathrm{s}}{\longrightarrow}$ to denote convergence in the mean-squared $\left(L^{2}\right)$ sense [31].

${ }^{7}$ A similar lemma exists for matrices $\boldsymbol{S}^{*} \boldsymbol{S}$ where the $N$-dimensional columns of $S$ are independent and drawn uniformly from the surface of the unit $N$-sphere.
} 
ments of $\boldsymbol{S}$ being independent $\mathcal{C N}\left(0, \sigma^{2}\right)$. The lemma and its proof rely on the concepts of isotropically distributed vectors and matrices, which are reviewed in [12, Appendix A].

Lemma 1: Let the elements of an $N \times M$ matrix $\boldsymbol{S}$ be independent $\mathcal{C N}\left(0, \sigma^{2}\right)$. Then, the eigenvector matrix of $\boldsymbol{S}^{*} \boldsymbol{S}$ is isotropically distributed unitary and independent of the eigenvalues.

To prove Theorem 4, we need to determine the limits of $\left[\left(\boldsymbol{S}^{*} \boldsymbol{S}\right)^{1 / 2}\right]_{m m},\left[P_{\mathcal{V}}\right]_{m m}$, and $\left[\boldsymbol{S}^{*} \boldsymbol{S}\right]_{m m}$ as $M \rightarrow \infty$ with $\beta$ held constant.

Let $\boldsymbol{S}=\boldsymbol{U} \boldsymbol{\Sigma} \boldsymbol{R}^{*}$ be the SVD of $\boldsymbol{S}$, where $\boldsymbol{U}$ is an $N \times N$ unitary matrix, $\boldsymbol{R}$ is an $M \times M$ unitary matrix, and $\boldsymbol{\Sigma}$ is a diagonal $N \times M$ matrix with diagonal elements $\sigma_{i} \geq 0$. Then, the quantity $\left[\left(\boldsymbol{S}^{*} \boldsymbol{S}\right)^{1 / 2}\right]_{\mathrm{mm}}$ can be written as

$$
\left[\left(\boldsymbol{S}^{*} \boldsymbol{S}\right)^{1 / 2}\right]_{m m}=\left[\boldsymbol{R} \boldsymbol{\Lambda}^{1 / 2} \boldsymbol{R}^{*}\right]_{m m}=\boldsymbol{y}_{m}^{*} \boldsymbol{\Lambda}^{1 / 2} \boldsymbol{y}_{m}
$$

where $\boldsymbol{y}_{m}$ is the $m$ th column of $\boldsymbol{R}^{*}$. Now, $\boldsymbol{R}$ and $\boldsymbol{\Lambda}$ are the eigenvector matrix and the eigenvalue matrix, respectively, in the eigendecomposition of the Wishart matrix $\boldsymbol{S}^{*} \boldsymbol{S}$. Thus, using Lemma 1 , we conclude that $\boldsymbol{R}^{*}$ is an isotropically distributed unitary matrix independent of $\boldsymbol{\Lambda}$. Since $\boldsymbol{y}_{m}$ is a column of an isotropically distributed unitary matrix, $\boldsymbol{y}_{m}$ is an isotropically distributed unit vector [33]. Consequently, $\boldsymbol{y}_{m}$ has the same distribution as $\boldsymbol{z} / \sqrt{\boldsymbol{z}^{*} \boldsymbol{z}}$, where $\boldsymbol{z}$ is an $M$-dimensional vector of independent $\mathcal{C} \mathcal{N}(0,1)$ random variables. Thus, $\left[\left(\boldsymbol{S}^{*} \boldsymbol{S}\right)^{1 / 2}\right]_{m m}$ has the same distribution as

$$
\frac{\boldsymbol{z}^{*} \boldsymbol{\Lambda}^{1 / 2} \boldsymbol{z}}{\boldsymbol{z}^{*} \boldsymbol{z}}=\frac{\sum_{j=1}^{M} \sqrt{\lambda_{j}}\left|z_{j}\right|^{2} / M}{\sum_{j=1}^{M}\left|z_{j}\right|^{2} / M}
$$

with the $\lambda_{j}$ 's denoting the eigenvalues of $\boldsymbol{S}^{*} \boldsymbol{S}$ and the $z_{j}$ 's denoting the components of $\boldsymbol{z}$. To evaluate the limit of (55) when $M \rightarrow \infty$, we rely on the following pair of lemmas.

Lemma 2 [34]: If the ratio of the number of users to the signature length is, or converges to, a constant

$$
\lim _{M \rightarrow \infty} \frac{M}{N}=\beta \in(0, \infty)
$$

then the percentage of the $M$ eigenvalues of $\boldsymbol{S}^{*} \boldsymbol{S}$ that lie below $x$ converges to the cumulative distribution function of the probability density function

$$
f_{\beta}(x)=\left[1-\beta^{-1}\right]^{+} \delta(x)+\frac{\sqrt{\left[x-\eta_{1}\right]^{+}\left[\eta_{2}-x\right]^{+}}}{2 \pi \beta x} .
$$

Here, $\eta_{1}$ and $\eta_{2}$ are defined according to (52) and (53), and the operator $[\cdot]^{+}$is defined according to

$$
[u]^{+} \triangleq \max \{0, u\}
$$

Lemma 3 [12]: Let $\left\{c_{j}\right\}$ denote a set of independent and identically distributed (i.i.d.) random variables independent of $\left\{\lambda_{j}\right\}$ with $E\left(\left(c_{1}-E\left(c_{1}\right)\right)^{2}\right)<\infty$, where $\left\{\lambda_{j}\right\}$ denote the eigenvalues of a Wishart matrix under the conditions of Lemma 2. Furthermore, let $g(\cdot)$ be a function such that $g(0)<\infty$ and $g(x)<\infty$ for $x \in\left[(1-\sqrt{\beta})^{2},(1+\sqrt{\beta})^{2}\right]$, and such that
$E\left(g^{2}\left(\lambda_{1}\right)\right)<\infty$ when evaluated according to the probability density function $f_{\beta}(x)$ of (57). Then, as $M \rightarrow \infty$

$$
\frac{1}{M} \sum_{j=1}^{M} g\left(\lambda_{j}\right) c_{j} \stackrel{\text { m.s. }}{\longrightarrow} E\left(g\left(\lambda_{1}\right)\right) E\left(c_{1}\right)
$$

where $E\left(g\left(\lambda_{1}\right)\right)$ is evaluated according to $f_{\beta}(x)$.

Applying Lemma 3 and the strong law of large numbers to the numerator and denominator of (55), respectively, and noting that in this case $g(x)=\sqrt{x}$ so that $g(x)$ satisfies the conditions of Lemma 3, we have

$$
\left[\left(\boldsymbol{S}^{*} \boldsymbol{S}\right)^{1 / 2}\right]_{m m} \stackrel{\text { m.s. }}{\longrightarrow} \frac{E\left(\sqrt{\lambda_{1}}\right) E\left(\left|z_{1}\right|^{2}\right)}{E\left(\left|z_{1}\right|^{2}\right)}=E\left(\sqrt{\lambda_{1}}\right)
$$

as $M \rightarrow \infty$, where $E\left(\sqrt{\lambda_{1}}\right)$ is evaluated according to the probability density function $f_{\beta}(x)$ of (57). Thus,

$$
\begin{aligned}
& E\left(\sqrt{\lambda_{1}}\right)= \int_{0}^{\infty} \sqrt{x} f_{\beta}(x) d x \\
&= \int_{0}^{\infty} \sqrt{x}\left\{\left[1-\beta^{-1}\right]^{+} \delta(x)\right. \\
&\left.+\frac{\sqrt{\left[x-\eta_{1}\right]^{+}\left[\eta_{2}-x\right]^{+}}}{2 \pi \beta x}\right\} d x \\
&= \int_{\eta_{1}}^{\eta_{2}} \frac{\sqrt{\left(x-\eta_{1}\right)\left(\eta_{2}-x\right)}}{2 \pi \beta \sqrt{x}} d x \\
&= \frac{\sqrt{\eta_{2}}}{3 \pi \beta}\left[\left(\eta_{1}+\eta_{2}\right) E\left(\sqrt{1-\eta_{1} / \eta_{2}}\right)\right. \\
&\left.-2 \eta_{1} K\left(\sqrt{1-\eta_{1} / \eta_{2}}\right)\right]
\end{aligned}
$$

where the last equality is from [32], and where $K(\cdot), E(\cdot), \eta_{1}$, and $\eta_{2}$ are defined by (50), (51), (52), and (53), respectively.

Similarly, from $(6),[P \mathcal{V}]_{m m}$ can be written as

$$
[P \mathcal{V}]_{m m}=\left[\boldsymbol{R} \tilde{\boldsymbol{I}} \boldsymbol{R}^{*}\right]_{m m}=\boldsymbol{y}_{m}^{*} \tilde{\boldsymbol{I}} \boldsymbol{y}_{m}
$$

which has the same distribution as

$$
\frac{\boldsymbol{z}^{*} \tilde{\boldsymbol{I}} \boldsymbol{z}}{\boldsymbol{z}^{*} \boldsymbol{z}}=\frac{\sum_{j=1}^{M} \mu_{j}\left|z_{j}\right|^{2} / M}{\sum_{j=1}^{M}\left|z_{j}\right|^{2} / M}
$$

where

$$
\mu_{j}= \begin{cases}1, & \lambda_{j} \neq 0 \\ 0, & \lambda_{j}=0 .\end{cases}
$$

Applying Lemma 3 and the strong law of large numbers to the numerator and denominator of (63), respectively, we have

$$
[P \mathcal{V}]_{m m} \stackrel{\text { m.s. }}{\longrightarrow} \frac{E\left(\mu_{1}\right) E\left(\left|z_{1}\right|^{2}\right)}{E\left(\left|z_{1}\right|^{2}\right)}=E\left(\mu_{1}\right)
$$

as $M \rightarrow \infty$, where $E\left(\mu_{1}\right)$ is evaluated according to the probability density function $f_{\beta}(x)$ of (57). Thus,

$$
\begin{aligned}
{\left[P_{\mathcal{V}}\right]_{m m} \stackrel{\text { m.s. }}{\longrightarrow} E\left(\mu_{1}\right) } & =\lim _{M \rightarrow \infty} P\left(\lambda_{1} \neq 0\right) \\
& = \begin{cases}1, & \beta \leq 1 \\
\frac{1}{\beta}, & \beta>1 .\end{cases}
\end{aligned}
$$




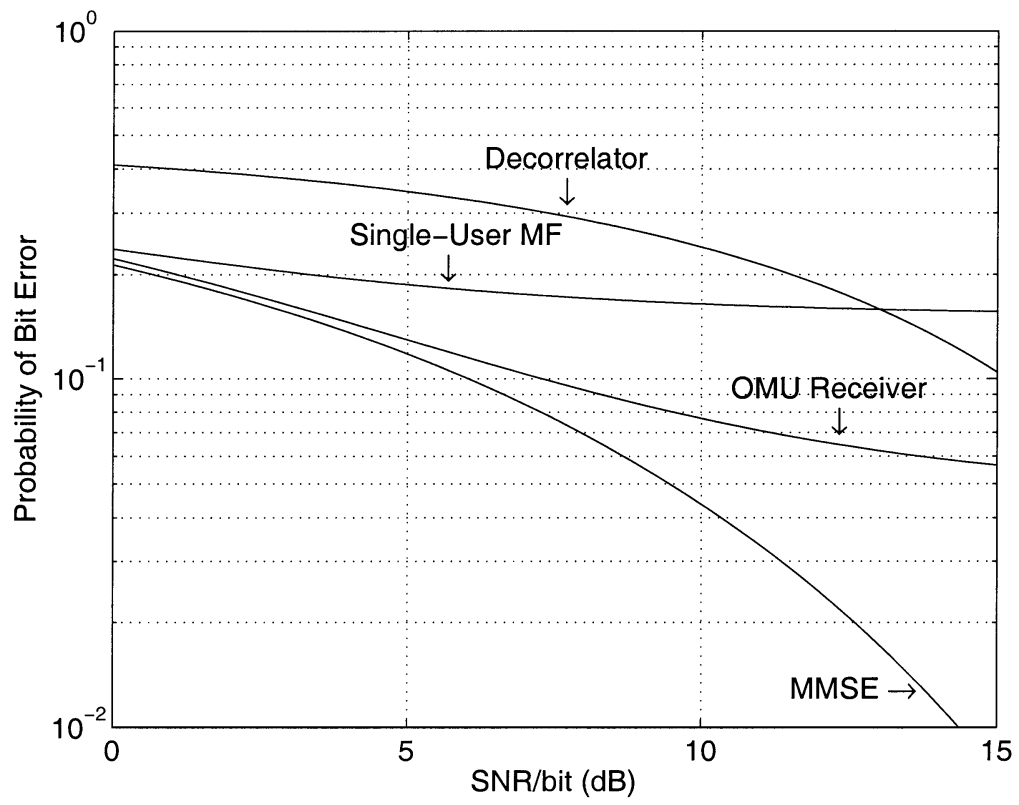

Fig. 8. Probability of bit error in the large-system limit, with equal-power users, random signatures, and $\beta=0.95$.

Finally,

$$
\left[\boldsymbol{S}^{*} \boldsymbol{S}\right]_{m m}=\boldsymbol{s}_{m}^{*} \boldsymbol{s}_{m}=\sum_{j=1}^{N} s_{j m}^{2} \stackrel{\text { m.s. }}{\longrightarrow} 1
$$

by the strong law of large numbers, with the $s_{j m}$ 's denoting the components of $\boldsymbol{s}_{m}$.

It is well known that if $x_{n} \stackrel{\text { m.s. }}{\longrightarrow} \bar{x}$ and $y_{n} \stackrel{\text { m.s. }}{\longrightarrow} \bar{y}$, then $x_{n} \pm$ $y_{n} \stackrel{\text { m.s. }}{\longrightarrow} \bar{x} \pm \bar{y}$ and $x_{n} y_{n} \stackrel{\text { m.s. }}{\longrightarrow} \overline{x y}$ [31]. The following lemma which involves the convergence of $1 / x_{n}$ is now required to complete the proof of Theorem 4 .

Lemma 4 [12]: Let $x_{n} \stackrel{\text { m.s. }}{\longrightarrow} \bar{x}$, where $\left\{x_{n}\right\}$ is a sequence of random variables such that $\left|1 / x_{n}\right| \leq B$ for all $n$, and $\bar{x} \neq 0$. Then

$$
\frac{1}{x_{n}} \stackrel{\text { m.s. }}{\longrightarrow} \frac{1}{\bar{x}} \text {. }
$$

Substituting (61), (66), and (67) into (46), and using the fact that $\gamma_{m} \leq 1 / \zeta$ with Lemma 4 completes the proof of Theorem 4 .

Since the MAI is asymptotically Gaussian in the infinite-user limit, we expect (47) to be an accurate approximation to the bit-error rate at all SNR, where $\gamma_{m}$ is given by Theorem 4 . We will use this approximation to compute the bit-error rate for the remainder of this section.

In Fig. 8, the bit-error rate in the infinite-user limit for the OMU receiver is compared to the single-user MF, the decorrelator, and the linear MMSE receiver, for $\beta=0.95$. For the SNR range shown, the OMU receiver performs better than the decorrelator and the MF. At low SNR, the performance of the OMU receiver is close to that of the linear MMSE receiver. In Fig. 9, we plot the probability of bit error ${ }^{8}$ in the infinite-user limit as a

\footnotetext{
${ }^{8}$ The asymptotic large system performance of the decorrelator for the case $\beta>1$ is derived in [12].
}

function of $\beta$, with an SNR of $8 \mathrm{~dB}$. For $\beta$ roughly greater than 0.6 but less than 1.4 , the OMU receiver performs significantly better than both the decorrelator and the MF.

\section{CONCLUSION}

In this paper, we developed a new multiuser receiver for CDMA channels, referred to as the OMU receiver, which relies on knowledge of only the signature vectors to mitigate the effect of both MAI and additive noise. First, we showed that the receiver can be interpreted as a decorrelator receiver followed by an MMSE whitening transformation that optimally whitens the output of the decorrelator prior to detection, so that it compensates for the noise enhancement of the decorrelator receiver without reintroducing too much MAI. In fact, we showed that for most practical cases of signature vectors, this receiver minimizes the MAI among all receivers that result in uncorrelated outputs. Second, we expressed this receiver as a correlation demodulator whose correlating vectors are orthonormal in the case of linearly independent signature vectors or form a normalized tight frame for the space spanned by the signature vectors in the case of linearly dependent signatures, and, furthermore, are closest in a least squares sense to the signatures and to the decorrelator vectors. Third, we demonstrated that the OMU receiver is also equivalent to an MF receiver followed by an MMSE whitening transformation. This new receiver can be implemented efficiently using any of the well-known algorithms for computing the SVD or the polar decomposition.

Finally, we developed tools to analyze the large-system performance of this receiver. Our analysis demonstrates that without knowledge of channel parameters, the OMU receiver in many cases results in improved performance over both the decorrelator and the MF receivers, particularly over practical ranges of SNR. 


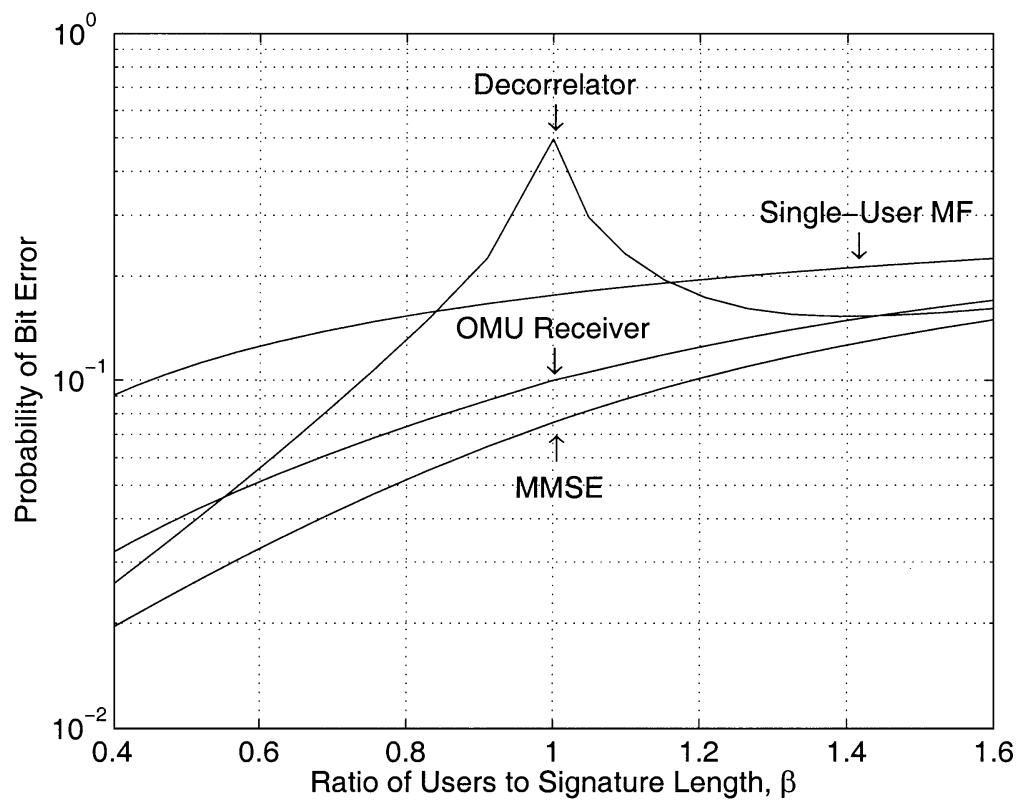

Fig. 9. Probability of bit error as a function of $\beta$ in the large-system limit, with equal-power users, random signatures, and SNR of $8 \mathrm{~dB}$.

\section{APPENDIX}

In this appendix we prove Theorem 2.

Let $x_{m}$ denote the $m$ th output of the demodulator of Fig. 2, where $\boldsymbol{B}=\boldsymbol{W}$ is an arbitrary whitening transformation. We may express $x_{m}$ as the $m$ th output of a bank of correlators with vectors $\boldsymbol{g}_{m}$ that are columns of $\boldsymbol{G}=\boldsymbol{V} \boldsymbol{W}^{*}$, where the vectors $\boldsymbol{g}_{m}$ form a normalized tight frame for $\mathcal{U}$. Let $a_{m}=\boldsymbol{v}_{m}^{*} \boldsymbol{r}$ denote the $m$ th output of the decorrelator demodulator. Then $a_{m}^{\prime}=\boldsymbol{v}_{m}^{*} \boldsymbol{n}$, $x_{m}^{\prime}=\boldsymbol{g}_{m}^{*} \boldsymbol{n}$, and

$$
\begin{aligned}
\sum_{m=1}^{M} E\left(\left(a_{m}^{\prime}-x_{m}^{\prime}\right)^{2}\right) & =\sum_{m=1}^{M} E\left(\left(\boldsymbol{v}_{m}-\boldsymbol{g}_{m}\right)^{*} \boldsymbol{n} \boldsymbol{n}^{*}\left(\boldsymbol{v}_{m}-\boldsymbol{g}_{m}\right)\right) \\
& =\sigma^{2} \sum_{m=1}^{M}\left(\boldsymbol{v}_{m}-\boldsymbol{g}_{m}\right)^{*}\left(\boldsymbol{v}_{m}-\boldsymbol{g}_{m}\right) .
\end{aligned}
$$

From (69), we conclude that minimizing the MSE defined by (5) is equivalent to minimizing the least squares error $\varepsilon_{\mathrm{LS}}\left(\left\{\boldsymbol{v}_{m}\right\},\left\{\boldsymbol{g}_{m}\right\}\right)$.

We now show that the OMU vectors also form the closest normalized tight frame to the signature vectors $\boldsymbol{s}_{m}$. In other words, the vectors $\boldsymbol{g}_{m}$ that minimize $\varepsilon_{\mathrm{LS}}\left(\left\{\boldsymbol{v}_{m}\right\},\left\{\boldsymbol{g}_{m}\right\}\right)$ and $\varepsilon_{\mathrm{LS}}\left(\left\{\boldsymbol{s}_{m}\right\},\left\{\boldsymbol{g}_{m}\right\}\right)$ are equal, subject to the constraint

$$
\boldsymbol{G}^{*} \boldsymbol{G}=P \mathcal{V}
$$

where $\boldsymbol{G}$ is the matrix of columns $\boldsymbol{g}_{m}$ and $\mathcal{V}=\mathcal{N}(\boldsymbol{G})^{\perp}$ is a $K$-dimensional space. To this end we rely on the following lemmas.

Lemma 5: Let $\left\{\boldsymbol{y}_{m} \in \mathbb{C}^{N}, 1 \leq m \leq M\right\}$ be a set of vectors where the first $K$ vectors are orthogonal, i.e.,

$$
\boldsymbol{y}_{k}^{*} \boldsymbol{y}_{m}=c_{m}^{2} \delta_{k m}, \quad 1 \leq k, m \leq K
$$

with $c_{m}>0$, and the remaining vectors are equal to 0 , i.e., $\boldsymbol{y}_{m}=0$ for $K+1 \leq m \leq M$.Then, the normalized tight frame that minimizes $\varepsilon_{\mathrm{LS}}\left(\left\{\boldsymbol{y}_{m}\right\},\left\{\boldsymbol{g}_{m}\right\}\right)$ is $\boldsymbol{g}_{m}=\boldsymbol{y}_{m} / c_{m}$ for $1 \leq m \leq K$, and $\boldsymbol{g}_{m}=0$ for $K+1 \leq m \leq M$.
Proof: First we show that minimizing $\varepsilon_{\mathrm{LS}}\left(\left\{\boldsymbol{y}_{m}\right\},\left\{\boldsymbol{g}_{m}\right\}\right)$ subject to (70) is equivalent to maximizing $\sum_{m=1}^{M} \Re\left\{\boldsymbol{g}_{m}^{*} \boldsymbol{y}_{m}\right\}$ subject to this constraint, where $\Re\{\cdot\}$ denotes the real part of the corresponding variable.

Expanding $\varepsilon_{\mathrm{LS}}\left(\left\{\boldsymbol{y}_{m}\right\},\left\{\boldsymbol{g}_{m}\right\}\right)$ we have

$$
\begin{aligned}
\varepsilon_{\mathrm{LS}}\left(\left\{\boldsymbol{y}_{m}\right\},\left\{\boldsymbol{g}_{m}\right\}\right) & =\sum_{m=1}^{M}\left(\boldsymbol{y}_{m}-\boldsymbol{g}_{m}\right)^{*}\left(\boldsymbol{y}_{m}-\boldsymbol{g}_{m}\right) \\
& =\sum_{m=1}^{M}\left(1+\boldsymbol{g}_{m}^{*} \boldsymbol{g}_{m}-2 \Re\left\{\boldsymbol{y}_{m}^{*} \boldsymbol{g}_{m}\right\}\right) .
\end{aligned}
$$

Since the vectors $\left\{\boldsymbol{g}_{m}, 1 \leq m \leq M\right\}$ form a normalized tight frame for a $K$-dimensional space $\mathcal{U} \subseteq \mathbb{C}^{N}, \boldsymbol{g}_{m}=P_{\mathcal{U}} \boldsymbol{h}_{m}$ for some orthonormal set of vectors $\left\{\boldsymbol{h}_{m}, 1 \leq m \leq M\right\}$ that span an $M$-dimensional subspace $\mathcal{W} \subseteq \mathbb{C}^{N}$ containing $\mathcal{U}$. Let $G$ and $\boldsymbol{H}$ denote the matrices of columns $\boldsymbol{g}_{m}$ and $\boldsymbol{h}_{m}$, respectively. Then, $\boldsymbol{G}=P_{\mathcal{U}} \boldsymbol{H}$ and

$$
\sum_{m=1}^{M} \boldsymbol{g}_{m}^{*} \boldsymbol{g}_{m}=\operatorname{tr}\left(\boldsymbol{G}^{*} \boldsymbol{G}\right)=\operatorname{tr}\left(\boldsymbol{H}^{*} P_{\mathcal{U}} \boldsymbol{H}\right)
$$

Since the columns of $\boldsymbol{H}$ are orthonormal and $\operatorname{span} \mathcal{W}$, we can express $P_{\mathcal{W}}$ as

$$
P_{\mathcal{W}}=\boldsymbol{H} \boldsymbol{H}^{*}
$$

Substituting (74) into (73) we have

$$
\sum_{m=1}^{M} \boldsymbol{g}_{m}^{*} \boldsymbol{g}_{m}=\operatorname{tr}\left(P_{\mathcal{W}} P_{\mathcal{U}}\right)=\operatorname{tr}\left(P_{\mathcal{U}}\right)=K
$$

where we used the fact that $P_{\mathcal{W}} P_{\mathcal{U}}=P_{\mathcal{U}}$ since $\mathcal{U} \subset \mathcal{W}$, and $\operatorname{tr}\left(P_{\mathcal{U}}\right)$ is equal to the dimension of $\mathcal{U}$. Combining (75) with (72) we conclude that minimizing $\varepsilon_{\mathrm{LS}}\left(\left\{\boldsymbol{y}_{m}\right\},\left\{\boldsymbol{g}_{m}\right\}\right)$ is equivalent to maximizing $\sum_{m=1}^{M} \Re\left\{\boldsymbol{y}_{m}^{*} \boldsymbol{g}_{m}\right\}$. 
Next, using the Chauchy-Schwarz inequality

$$
\begin{aligned}
\sum_{m=1}^{M} \Re\left\{\boldsymbol{g}_{m}^{*} \boldsymbol{y}_{m}\right\} & =\sum_{m=1}^{K} \Re\left\{\boldsymbol{g}_{m}^{*} \boldsymbol{y}_{m}\right\} \\
& \leq \sum_{m=1}^{K}\left|\boldsymbol{g}_{m}^{*} \boldsymbol{y}_{m}\right| \\
& \leq \sum_{m=1}^{K}\left(\boldsymbol{y}_{m}^{*} \boldsymbol{y}_{m}\right)^{1 / 2}\left(\boldsymbol{g}_{m}^{*} \boldsymbol{g}_{m}\right)^{1 / 2} \\
& \leq \sum_{m=1}^{K}\left(\boldsymbol{y}_{m}^{*} \boldsymbol{y}_{m}\right)^{1 / 2}
\end{aligned}
$$

where we used the fact that $\boldsymbol{g}_{m}^{*} \boldsymbol{g}_{m} \leq 1$, since $\boldsymbol{g}_{m}$ is an orthogonal projection of a normalized vector. We have equality in (76) if and only if $\boldsymbol{g}_{m}=\boldsymbol{y}_{m} d_{m}, 1 \leq m \leq K$ for some $d_{m}>0$, and $\boldsymbol{g}_{m}^{*} \boldsymbol{g}_{m}=1,1 \leq k \leq K$. In addition, to satisfy (70), we must have that $G^{*} \boldsymbol{G}$ is a rank- $K$ orthogonal projection operator, where $\boldsymbol{G}$ is the matrix of columns $\boldsymbol{g}_{m}$. Thus, $\boldsymbol{g}_{m}=\boldsymbol{y}_{m} / c_{m}$, $1 \leq m \leq K$, and $\boldsymbol{g}_{m}=0, K+1 \leq m \leq M$.

As a result of Lemma 5, it follows that if we have two sets of orthogonal vectors, where each vector from one set is proportional to a vector in the other set, then the closest normalized tight frame to each one of these sets of vectors is the same.

Lemma 6: Let the columns of $\boldsymbol{G}$ be the normalized tight frame vectors $\boldsymbol{g}_{m}$ that minimize $\varepsilon_{\mathrm{LS}}\left(\left\{\boldsymbol{v}_{m}\right\},\left\{\boldsymbol{g}_{m}\right\}\right)$, and let $\overline{\boldsymbol{v}}_{m}$ denote the columns of $\overline{\boldsymbol{V}}=\boldsymbol{V} \boldsymbol{U}$, where $\boldsymbol{U}$ is an arbitrary unitary matrix. Then the normalized tight frame vectors $\overline{\boldsymbol{g}}_{m}$ that minimize $\varepsilon_{\mathrm{LS}}\left(\left\{\overline{\boldsymbol{v}}_{m}\right\},\left\{\overline{\boldsymbol{g}}_{m}\right\}\right)$ are the columns of $\overline{\boldsymbol{G}}=\boldsymbol{G U}$.

Proof: For any normalized tight frame $\boldsymbol{g}_{m}$ that are the columns of $\boldsymbol{G}$, we have

$$
\begin{aligned}
\varepsilon_{\mathrm{LS}}\left(\left\{\boldsymbol{v}_{m}\right\},\left\{\boldsymbol{g}_{m}\right\}\right) & =\operatorname{tr}\left((\boldsymbol{V}-\boldsymbol{G})^{*}(\boldsymbol{V}-\boldsymbol{G})\right) \\
& =\operatorname{tr}\left(\boldsymbol{U}^{*}(\boldsymbol{V}-\boldsymbol{G})^{*}(\boldsymbol{V}-\boldsymbol{G}) \boldsymbol{U}\right) \\
& =\operatorname{tr}\left((\overline{\boldsymbol{V}}-\boldsymbol{G} \boldsymbol{U})^{*}(\overline{\boldsymbol{V}}-\boldsymbol{G} \boldsymbol{U})\right) \\
& =\varepsilon_{\mathrm{LS}}\left(\left\{\overline{\boldsymbol{v}}_{m}\right\},\left\{\overline{\boldsymbol{g}}_{m}\right\}\right)
\end{aligned}
$$

where $\overline{\boldsymbol{g}}_{m}$ are the columns of $\overline{\boldsymbol{G}}=\boldsymbol{G} \boldsymbol{U}$. Furthermore, the vectors $\overline{\boldsymbol{g}}_{m}$ also form a normalized tight frame. Indeed, since the vectors $\boldsymbol{g}_{m}$ form a normalized tight frame, $\boldsymbol{G} \boldsymbol{G}^{*}=P_{\mathcal{R}(\boldsymbol{G})}$. But,

$$
\overline{G G}^{*}=G U U^{*} G^{*}=G G^{*}
$$

and, consequently, the vectors $\overline{\boldsymbol{g}}_{m}$ also form a normalized tight frame.

From Lemmas 5 and 6 it follows that if we can find a unitary matrix such that the first $K$ columns $\overline{\boldsymbol{v}}_{m}$ of $\overline{\boldsymbol{V}}=\boldsymbol{V} \boldsymbol{U}$ are orthogonal and the remaining columns are all 0 , the first $K$ columns $\overline{\boldsymbol{s}}_{m}$ of $\overline{\boldsymbol{S}}=\boldsymbol{S} \boldsymbol{U}$ are orthogonal and the remaining columns are all 0 , and $\overline{\boldsymbol{v}}_{m}$ is proportional to $\overline{\boldsymbol{s}}_{m}$, then the normalized tight frame minimizing $\varepsilon_{\mathrm{LS}}\left(\left\{\boldsymbol{v}_{m}\right\},\left\{\boldsymbol{g}_{m}\right\}\right)$ and the normalized tight frame minimizing $\varepsilon_{\mathrm{LS}}\left(\left\{\boldsymbol{s}_{m}\right\},\left\{\boldsymbol{g}_{m}\right\}\right)$ are the same.

We now show that such a unitary matrix exists. Let $S=$ $\boldsymbol{U} \boldsymbol{\Sigma} \boldsymbol{R}^{*}$ be the SVD of $\boldsymbol{S}$, where $\boldsymbol{U}$ and $\boldsymbol{R}$ are unitary matrices and $\boldsymbol{\Sigma}$ is a diagonal $N \times M$ matrix with diagonal elements $\sigma_{m}>0,1 \leq m \leq K$, and 0 otherwise. Then

$$
V=S\left(S^{*} S\right)^{\dagger}=U \tilde{\Sigma} R^{*}
$$

where $\tilde{\Sigma}$ is a diagonal $N \times M$ matrix with diagonal elements $1 / \sigma_{m}, 1 \leq m \leq K$ and 0 otherwise. Now, let $\overline{\boldsymbol{V}}=\boldsymbol{V} \boldsymbol{R}=\boldsymbol{U} \tilde{\boldsymbol{\Sigma}}$ and $\overline{\boldsymbol{S}}=\boldsymbol{S} \boldsymbol{R}=\boldsymbol{U} \boldsymbol{\Sigma}$. Then the first $K$ columns $\overline{\boldsymbol{v}}_{m}$ of $\overline{\boldsymbol{V}}$ are orthogonal, and the remaining columns are all 0. Similarly, the first $K$ columns $\overline{\boldsymbol{s}}_{m}$ of $\overline{\boldsymbol{S}}$ are orthogonal, and the remaining columns are all 0 . Furthermore, $\overline{\boldsymbol{v}}_{m}=\overline{\boldsymbol{s}}_{m} / \sigma_{m}^{2}$ for $1 \leq m \leq K$. Thus, the normalized tight frame minimizing $\varepsilon_{\mathrm{LS}}\left(\left\{\boldsymbol{v}_{m}\right\},\left\{\boldsymbol{g}_{m}\right\}\right)$ is the same as the normalized tight frame minimizing $\varepsilon_{\mathrm{LS}}\left(\left\{\boldsymbol{s}_{m}\right\},\left\{\boldsymbol{g}_{m}\right\}\right)$.

\section{ACKNOWLEDGMENT}

The authors wish to thank Prof. A. V. Oppenheim and M. J. Lopez for many fruitful discussions.

\section{REFERENCES}

[1] S. Verdú, Multiuser Detection. New York: Cambridge Univ. Press, 1998.

[2] Y. C. Eldar and A. V. Oppenheim, "Orthogonal multiuser detection," Signal Processing, vol. 82, pp. 321-325, Feb. 2002.

[3] Y. C. Eldar, "Quantum signal processing," Ph.D. dissertation, MIT, Cambridge, MA, Dec. 2001.

[4] Y. C. Eldar and A. V. Oppenheim, "MMSE whitening and subspace whitening," IEEE Trans. Inform. Theory, vol. 49, pp. 1846-1851, July 2003.

[5] A. Duel-Hallen, "Decorrelating decision-feedback multiuser detector for synchronous code-division multiple-access channel," IEEE Trans. Commun., vol. 41, pp. 285-290, Feb. 1993.

[6] A. M. Monk, M. Davis, L. B. Milstein, and C. W. Helstrom, "A noisewhitening approach to multiple-access noise rejection-Part I: Theory and background," IEEE J. Select. Areas Commun., vol. 12, pp. 817-827, June 1994.

[7] Y. C. Yoon and H. Leib, "Matched filters with interference suppression capabilities for DS-CDMA," IEEE J. Select. Areas Commun., vol. 14, pp. 1510-1521, Oct. 1996.

[8] M. Rupf, F. Tarköy, and J. L. Massey, "User-separating demodulation for code-division multiple-access systems," IEEE J. Select. Areas Commun., vol. 12, pp. 786-795, June 1994.

[9] G. D. Forney, Jr., "Geometrically uniform codes," IEEE Trans. Inform. Theory, vol. 37, pp. 1241-1260, Sept. 1991.

[10] Y. C. Eldar and G. D. Forney, Jr., "On quantum detection and the square-root measurement," IEEE Trans. Inform. Theory, vol. 47, pp. 858-872, Mar. 2001.

[11] S. Ulukus and R. D. Yates, "Optimum multiuser detection is tractable for synchronous CDMA systems using $m$-sequences," IEEE Commun. Lett., vol. 2, pp. 89-91, Apr. 1998.

[12] Y. C. Eldar and A. M. Chan, "On the asymptotic performance of the decorrelator," IEEE Trans. Inform. Theory, vol. 49, pp. 2309-2313, Sept. 2003.

[13] D. N. C. Tse and S. V. Hanly, "Linear multiuser receivers: Effective interference, effective bandwidth and user capacity," IEEE Trans. Inform. Theory, vol. 45, pp. 641-657, Mar. 1999.

[14] S. Verdú and S. Shamai (Shitz), "Spectral efficiency of CDMA with random spreading," IEEE Trans. Inform. Theory, vol. 45, pp. 622-640, Mar. 1999.

[15] G. H. Golub and C. F. Van Loan, Matrix Computations, 3rd ed. Baltimore, MD: Johns Hopkins Univ. Press, 1996.

[16] R. Lupas and S. Verdú, "Linear multiuser detectors for synchronous code-division multiple-access channels," IEEE Trans. Inform. Theory, vol. 35, pp. 123-136, Jan. 1989.

[17] E. M. Friel and K. M. Pasala, "Direction finding with compensation for a near field scatterer," in Proc. Int. Symp. Antennas and Propagation Soc., 1995, pp. 106-109.

[18] R. J. Piechocki, N. Canagarajah, and J. P. McGeehan, "Improving the direction-of-arrival resolution via double code filtering in WCDMA," in Proc. 1st Int. Conf. $3 G$ Mobile Communication Technologies, Mar. 2000, pp. 204-207.

[19] R. A. Horn and C. R. Johnson, Matrix Analysis. Cambridge, U.K.: Cambridge Univ. Press, 1985.

[20] I. Daubechies, Ten Lectures on Wavelets. Philadelphia, PA: SIAM, 1992. 
[21] A. Aldroubi, "Portraits of frames," in Proc. Amer. Math. Soc., vol. 123, 1995, pp. 1661-1668.

[22] Y. C. Eldar and G. D. Forney, Jr., "Optimal tight frames and quantum measurement," IEEE Trans. Inform. Theory, vol. 48, pp. 599-610, Mar. 2002.

[23] Y. C. Eldar, "Least-squares inner product shaping," Linear Alg. Appl., vol. 348, pp. 153-174, May 2002.

[24] N. J. Higham, "Computing the polar decomposition-With applications," SIAM J. Sci. Stat. Comput., vol. 7, pp. 1160-1174, 1986.

[25] P. Zielinski and K. Zietak, "The polar decomposition-Properties, applications and algorithms," Matematyka Stosowana, vol. 38, pp. 23-40, 1995.

[26] C. W. Helstrom, "Bayes-cost reduction algorithm in quantum hypothesis testing," IEEE Trans. Inform. Theory, vol. 28, pp. 359-366, Mar. 1982.

[27] C. W. Helstrom, Quantum Detection and Estimation Theory. New York: Academic, 1976.

[28] Y. C. Eldar, A. Megretski, and G. C. Verghese. Optimal detection of symmetric mixed quantum states [Online]. Available: http://arxiv. org/abs/quant-ph/0211111; submitted for publication in IEEE Trans. Inform. Theory.
[29] Y. C. Eldar, A. Megretski, and G. C. Verghese, "Designing optimal quantum detectors via semidefinite programming," IEEE Trans. Inform. Theory, vol. 49, pp. 1007-1012, Apr. 2003.

[30] Y. C. Eldar, A. V. Oppenheim, and D. Egnor, "Orthogonal and projected orthogonal matched filter detection," Signal Processing, submitted for publication.

[31] K. L. Chung, A Course in Probability Theory. San Diego, CA: Academic, 2001.

[32] I. S. Gradshteyn and I. M. Ryzhik, Table of Integrals, Series and Products. New York: Academic, 1980.

[33] T. L. Marzetta and B. M. Hochwald, "Capacity of a mobile multiple-antenna communication link in Rayleigh flat fading," IEEE Trans. Inform. Theory, vol. 45, pp. 139-157, Jan. 1999.

[34] Z. D. Bai and Y. Q. Yin, "Limit of the smallest eigenvalue of a large dimensional sample covariance matrix," Ann. Probab., vol. 21, pp. 1275-1294, 1993. 